\title{
Eigenvalue topology optimization via efficient multilevel solution of the frequency
} response

Eigenvalue topology optimization

Ferrari, Federico; Lazarov, Boyan S.; Sigmund, Ole

Published in:

International Journal for Numerical Methods in Engineering

Link to article, DOI:

10.1002/nme.5829

Publication date:

2018

Document Version

Peer reviewed version

Link back to DTU Orbit

Citation (APA):

Ferrari, F., Lazarov, B. S., \& Sigmund, O. (2018). Eigenvalue topology optimization via efficient multilevel solution of the frequency response: Eigenvalue topology optimization. International Journal for Numerical Methods in Engineering, 115(7), 872-892. https://doi.org/10.1002/nme.5829

\section{General rights}

Copyright and moral rights for the publications made accessible in the public portal are retained by the authors and/or other copyright owners and it is a condition of accessing publications that users recognise and abide by the legal requirements associated with these rights.

- Users may download and print one copy of any publication from the public portal for the purpose of private study or research.

- You may not further distribute the material or use it for any profit-making activity or commercial gain

- You may freely distribute the URL identifying the publication in the public portal 


\title{
Eigenvalue topology optimization via efficient multilevel solution of the Frequency Response
}

\author{
Federico Ferrari ${ }^{\mathrm{a}, 1, *}$, Boyan S. Lazarov ${ }^{\mathrm{b}, 2}$, Ole Sigmund ${ }^{\mathrm{b}, 3}$ \\ ${ }^{a}$ Dipartimento di Ingegneria "Enzo Ferrari"-Università degli Studi di Modena e Reggio Emilia \\ ${ }^{b}$ Department of Mechanical Engineering, Solid Mechanics, DTU
}

\begin{abstract}
The article presents an efficient solution method for structural topology optimization aimed at maximizing the fundamental frequency of vibration. Nowadays this is still a challenging problem, mainly due to the high computational cost required by spectral analyses. The proposed method relies on replacing the eigenvalue problem with a frequency response one, which can be tuned and efficiently solved by a multilevel procedure. Connections of the method with multigrid eigenvalue solvers are discussed in details. Several applications demonstrating more than $90 \%$ savings of the computational time are presented as well.
\end{abstract}

Keywords: Topology optimization; Eigenvalue optimization; Fast solvers; Vibrations; Large-scale problems

\section{Introduction}

In this work we focus on the problem of maximizing the natural frequency of vibration of a structure by means of topology optimization 1, 2, 3, 4, 5. This classical problem finds important application in the design of engineering structures, whenever they must be protected against low frequency resonance [6, 7]. Within the context of a nested approach [ $[$, the solution of this problem by a gradient-based method requires the formulation and the direct solution of an eigenvalue problem at each optimization step, since the sensitivities depend on the eigenpairs 9, 10. This direct approach still presents some challenges compared to other simpler problems of topology optimization (e.g. the compliance problem). In particular, the following two difficulties can be pointed at.

First, eigenvalues are likely to become repeated, and the corresponding sensitivities non-smooth, during the optimization [11, 12. This theoretical difficulty has been extensively studied in the last decades and several methods have been proposed for computing sensitivities of repeated eigenvalues

\footnotetext{
${ }^{*}$ Corresponding author

Email addresses: federico.ferrari@unimore.it (Federico Ferrari), bslazarov@gmail.com (Boyan S. Lazarov), sigmund@mek.dtu.dk (Ole Sigmund)

${ }^{1} \mathrm{PhD}$ Student in Solid Mechanics

${ }^{2}$ Senior Researcher

${ }^{3}$ Full Professor of Solid Mechanics
} 
[13, 14, or for working with smooth functions of them [15, 16, 17. Other approaches overcome this issue by recurring to methods of semi-definite programming, or non-convex optimization [12, 18, 19. A second, practical limitation, is due to the high computational cost required by the solution of a large eigenvalue problem at each optimization step. Even with the availability of modern computer resources, this is still the main hurdle for bringing eigenvalue optimization to large scale.

To reduce the computational demand of the analysis, researchers have mainly resorted to approximate reanalysis procedures [20, 21, and in particular to the so-called Combined Approximations (CA), originally introduced for static reanalysis [22] and then extended to vibration problems 23. 24. CA have been applied to topology optimization, for the compliance problem [25], and also for free vibrations [26, obtaining remarkable computational savings. However, the direct solution of a full eigenvalue problem was still considered, even if only at few steps, and this may not be affordable for very large scale optimization problems.

Our aim is to set up a procedure which does not require the solution of any large scale eigenvalue problem. In 27] the foundations of such a method have been established, by surrogating the eigenvalue problem by a frequency response one, requiring the solution of linear systems only. It has been shown that, upon proper tuning of the harmonic excitation parameters, the frequency response surrogate approach gives essentially the same outcome as obtained for direct eigenvalue optimization. In particular, the amplitude of the harmonic load must be capable of exciting the fundamental mode and its driving frequency should be dynamically updated to stay close and, most important, below the first frequency peak. This last point is crucial to ensure the increase of the fundamental eigenvalue, and therefore a lower bound to its current value has to be determined.

Although the idea was proven to work, the computational procedure proposed in 27] left much space for improvings. First, the load amplitudes were defined in a rather heuristic way, barely generalizable, and only for the first optimization step, then kept fixed for the whole process. Second, the driving frequency was computed with a simple but potentially expensive procedure: a line search algorithm where several LU factorizations of the system matrix were needed. Last, the resulting frequency response problem was solved accurately by a direct method (Cholesky factorization), a prerogative that will be ruled out by the transition to large scale problems.

In this work we extend and enhance all these points, introducing a strategy based on multilevel discretizations. The general idea is to compute accurate eigenpairs only on the coarsest discretization, solving an eigenvalue problem way smaller than the original one. The coarse grid modes are projected and smoothened up to the finest scale, and here used as load vectors for the frequency response linear system. This is then solved by a conjugate gradient method, preconditioned by a multigrid cycle which is built on the same multilevel discretization. During the multilevel projection we can also compute upper and lower bounds to the fundamental eigenvalue. The latter, which is used for bounding the range of admissible driving frequencies, is now computed at the cost of solving an additional linear system. In this way, the overall method has multigrid efficiency, meaning that the computational effort scales almost linearly with the problem size 28 .

The remainder of the paper is organized as follows. In Section 2 the general setting and the formulation of the two optimization problems are given. In subsection 2.1 we shortly discuss the influence of the harmonic load parameters on the effectiveness of the surrogation. Section 3 is devoted to the description of the multilevel method, and in subsection 3.1 we also discuss its connection with some multigrid eigenvalue solvers. Numerical tests on 2D and 3D model problems are discussed in Section 5, demonstrating the effectiveness of the method and the computational savings achievable. Conclusions and some future perspectives are summarized in Section 6 . 
$3 \mathrm{~L}$

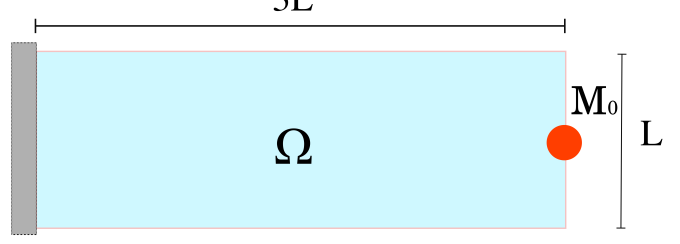

(a)

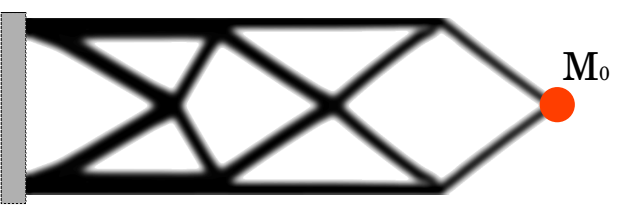

(b)

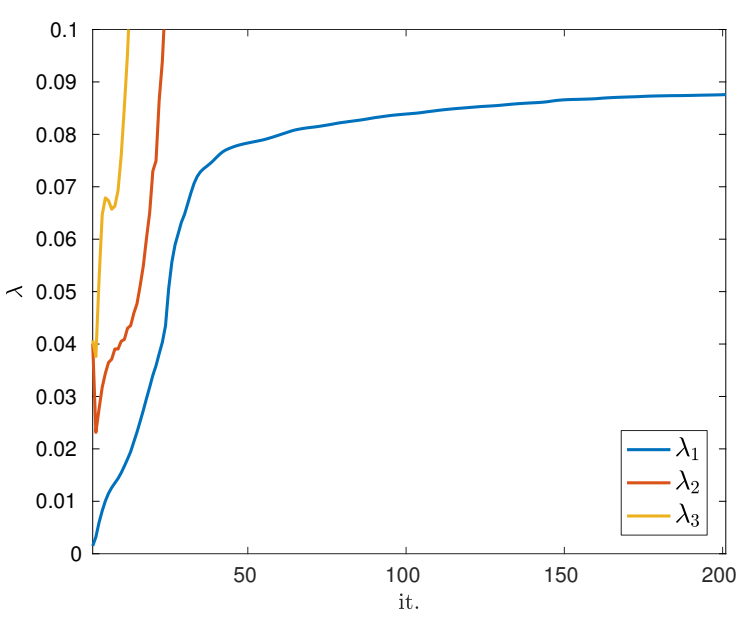

(c)

Figure 1: Geometrical description of the cantilever beam (a), optimized topology (b), and optimization history obtained by the direct solution of problem $\mathscr{P}$, defined by $2 \mathrm{a}-2 \mathrm{~b}$ (c)

\section{Formulation of the two optimization problems}

Let us refer to the cantilever depicted in Figure 1 (a), modeled with plane stress assumptions. We aim at maximizing its fundamental frequency of vibration through the optimal distribution of a given amount of material $V \leq V^{*}$ in $\Omega$. Following the standard approach of density-based topology optimization [1, $\Omega$ is discretized by a uniform and regular grid of $d$ finite elements, with characteristic size $h$, and to each element is assigned a design variable $x_{e} \in[0,1]$. Stiffness and density are related to the vector $x=\left\{x_{e}\right\}_{e=1}^{d}$ by the modified SIMP interpolation [29]

$$
E(x)=E_{0}+x^{p}\left(E_{1}-E_{0}\right), \quad \rho(x)=\rho_{0}+x\left(\rho_{1}-\rho_{0}\right)
$$

where $E_{1}$ and $\rho_{1}$ are the Young modulus and density of the solid material, $E_{0}$ and $\rho_{0}$ of the weak one and $p$ is a penalization parameter. The Poisson's ratio is independent of $x$ and set to $\nu=0.3$.

The problem under consideration can be formulated as

$$
\mathscr{P}\left\{\begin{array}{l}
\max _{x \in \mathcal{F}_{\text {ad }}} \min _{l=1, \ldots, n} \lambda_{l} \\
\text { st. } \quad K(x) \phi=\lambda\left(M_{s}(x)+M_{0}\right) \phi, \quad \phi \neq 0
\end{array}\right.
$$

where $\mathcal{F}_{\text {ad }}=\left\{x \in \mathbb{R}^{d} \mid \frac{h^{2}}{V^{*}} \sum_{e=1}^{d} x_{e}-1 \leq 0,0 \leq x_{e} \leq 1\right\}$ is the feasible set, $n$ is the total number of Degrees Of Freedom (DOFs) and $K(x)$ and $M_{s}(x)$ are the stiffness and structural mass matrices, symmetric and positive definite for all $x \in \mathcal{F}_{\text {ad }}$. The matrix $M_{0}$ accounts for non-structural masses and is symmetric and positive semi-definite. For the sake of brevity, the dependence on $x$ of $K$ and $M_{s}$ will be omitted and we will frequently refer to $M=M_{s}+M_{0}$.

With these assumptions, 2b has $n$ solutions $\left(\lambda_{i}, \phi_{i}\right)$, depending implicitly on $x$. The eigenvalues, proportional to the squared natural frequencies, can be ordered as $0 \leq \lambda_{1} \leq \lambda_{2} \leq \ldots \leq \lambda_{n}$. 


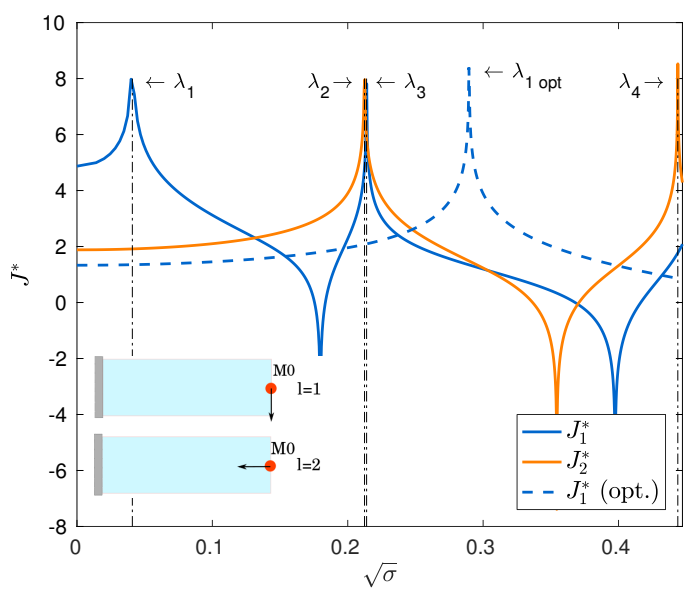

(a)

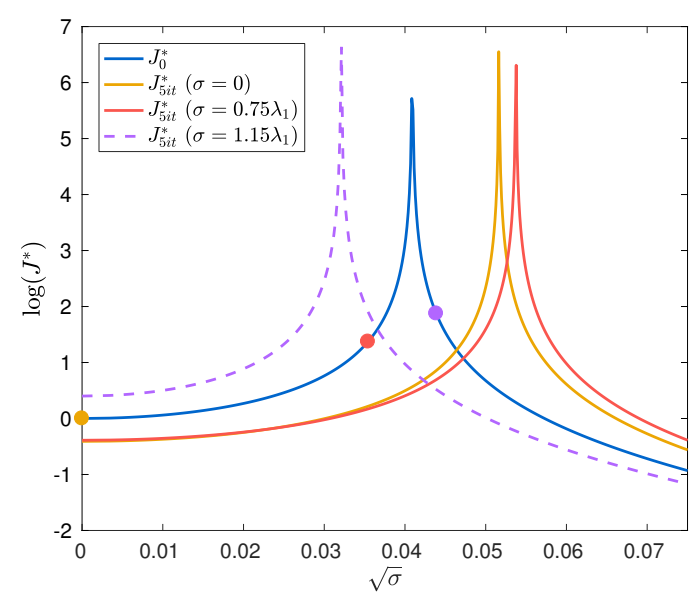

(b)

Figure 2: (a) shows the FRFs corresponding to two load cases, for the uniform cantilever structure (continuous curves) and for the optimized design of Figure 1 (b) (dashed curve). $\phi_{2}$ is an axial mode and therefore $\lambda_{2}$ is represented only in the FRF associated with the axial force. (b) shows the influence of the excitation frequency $\sigma$ on the shift of the first frequency peak induced by problem $\mathscr{P}_{*}$. Here, five optimization steps were considered for each case

The eigenvectors, representing the natural modes of vibration, fulfill the orthogonality relationships

$$
\phi_{i}^{T}\left(M_{s}+M_{0}\right) \phi_{j}=\delta_{i j}, \quad \phi_{i}^{T} K \phi_{j}=\lambda_{i} \delta_{i j}
$$

In Figure 1 (b)-(c) we see the optimization outcome for the particular parameters $L=1$, $V^{*}=0.3$ and $M_{0}=0.2 \rho V^{*}$.

Let us now consider a set of harmonic loads $\hat{f}_{l}=f_{l} e^{i \sqrt{\sigma} t}, l=1, \ldots, q$, defined by the amplitude vectors $f_{l}$ and the excitation frequency $\sqrt{\sigma}$, such that $\sigma>0$ is distinct from every eigenvalue. The harmonic response amplitudes $u_{l}$ are computed by solving the linear systems

$$
S(x ; \sigma) u_{l}=f_{l}, \quad l=1, \ldots, q
$$

where $S(x ; \sigma):=K-\sigma M$ is the dynamic stiffness matrix, which inherits the symmetry of $K$ and $M$ but is positive definite only for $\sigma<\lambda_{1}$. To each pair $\left(f_{l}, u_{l}\right)$ we can associate the functional

$$
J_{l}^{*}(x, \sigma):=\left|u_{l}^{T} f_{l}\right|=\left|u_{l}^{T} S(x ; \sigma) u_{l}\right|
$$

that is a measure of the so-called dynamic compliance of the structure subjected to $f_{l}$ [30] and, as a function of $\sigma$, also represents the system Frequency Response Function (FRF) [7]. Provided that a given load $f_{l}$ can excite the $l$-th mode, that is $f_{l}^{T} \phi_{l} \neq 0$, the FRF shows a peak at $\sigma=\lambda_{l}$, independently of the particular shape of $f_{l}$ (see Figure 2 (a)).

On the same feasible set we consider the problem of minimizing the FRF associated with the worst load case, at the specific location of the spectrum determined by $\sigma$

$$
\mathscr{P}_{*}\left\{\begin{array}{l}
\min _{x \in \mathcal{F}_{\text {ad }}} \max _{l=1, \ldots, q} J_{l}^{*}(x, \sigma) \\
\text { s.t. (4) }
\end{array}\right.
$$


As a side effect, the FRF peak nearest to $\sigma$ will move away from $\sigma$ itself, and this can be used to indirectly obtain a larger eigenvalue [3, 31, 27, as depicted in Figure 2 (b). To ensure the effectiveness of such an indirect effect, the harmonic parameters defining the load have to be properly selected and updated in the optimization proceeds, as discussed below.

\subsection{Selection of the harmonic load parameters}

Focusing first on the single mode case (i.e. $\lambda_{1}<\lambda_{2}$ ), we highlight the role of the harmonic parameters $f_{l}$ and $\sigma$ by expanding the response on the modal basis. Due to the relationships (3) the inverse of the dynamic stiffness matrix can be expressed as [7, 32

$$
S(x ; \sigma)^{-1}=\sum_{i=1}^{n} \frac{\phi_{i} \phi_{i}^{T}}{\phi_{i}^{T}(K-\sigma M) \phi_{i}}=\sum_{i=1}^{n} \frac{\phi_{i} \phi_{i}^{T}}{\lambda_{i}-\sigma}
$$

Without loss of generality we consider a mass proportional load $f_{l}=M \psi_{l}$, where $\psi_{l}$ is normalized as $\psi_{l}^{T} M \psi_{l}=1$. Introducing the modal forces $\gamma_{i l}=\phi_{i}^{T} M \psi_{l}$, such that $\sum_{i=1}^{n} \gamma_{i l}=1$, we can write

$$
u_{l}=S(x ; \sigma)^{-1} f_{l}=\sum_{i=1}^{n} \frac{\gamma_{i l}}{\lambda_{i}-\sigma} \phi_{i}
$$

Again, exploiting the orthogonality (3), the dynamic compliance (5) can be written as

$$
J_{l}^{*}=\sum_{i=1}^{n} \frac{\gamma_{i l}^{2}}{\left|\lambda_{i}-\sigma\right|}=\frac{\gamma_{1 l}^{2}}{\left|\lambda_{1}-\sigma\right|}+\varrho
$$

where in the rightmost expression we split the modal force associated with $\phi_{1}$ from the other terms, collected into a remainder $\varrho=\varrho\left(\gamma_{i l}\right), i>1$.

As long as the applied load has a dominant contribution on the mode associated with $\lambda_{1}, \gamma_{1 l}$ will be close to one, and from (8) we conclude that the minimization of $J_{l}^{*}$ is achieved mainly by increasing $\left|\lambda_{1}-\sigma\right|$. If we further fix $\sigma<\lambda_{1}$, this can only mean increasing $\lambda_{1}$ itself.

The derivative of (8) with respect to $x_{e}$ reads

$$
\frac{\partial J_{l}^{*}}{\partial x_{e}}=\sum_{i=1}^{n} \frac{2 \gamma_{i l}}{\left|\lambda_{i}-\sigma\right|} \frac{\partial \gamma_{i l}}{\partial x_{e}}-\frac{\operatorname{sign}\left(\lambda_{i}-\sigma\right) \gamma_{i l}^{2}}{\left|\lambda_{i}-\sigma\right|^{2}} \frac{\partial \lambda_{i}}{\partial x_{e}}
$$

and we can split it between a main term, proportional to $\gamma_{1 l}$, and a remainder $\tilde{\varrho}$

$$
\frac{\partial J_{l}^{*}}{\partial x_{e}}=-\frac{\operatorname{sign}\left(\lambda_{1}-\sigma\right) \gamma_{1 l}}{\left|\lambda_{1}-\sigma\right|^{2}} \frac{\partial \lambda_{1}}{\partial x_{e}}+\tilde{\varrho}
$$

Again, provided $\sigma<\lambda_{1}$, the descent direction for the dynamic compliance (that is $-\partial_{e} J_{l}^{*}$ ) contains, up to a scale factor, the increasing direction of the eigenvalue $\partial_{e} \lambda_{1}$, plus the contribution of the remainder term $\tilde{\varrho}$. Recalling the definition of the coefficients $\gamma_{i l}$, the latter reads

$$
\tilde{\varrho}=-\sum_{i=2}^{n} \frac{\operatorname{sign}\left(\lambda_{i}-\sigma\right) \gamma_{i l}^{2}}{\left|\lambda_{i}-\sigma\right|^{2}} \frac{\partial \lambda_{i}}{\partial x_{e}}+\sum_{i=1}^{n} \frac{\partial}{\partial x_{e}}\left(\psi^{T} M \phi_{i}\right)
$$

Eqs.(8) and (10) formalize the idea behind the surrogation of eigenvalue optimization, as defined by $\mathscr{P}$, with the frequency response minimization problem $\mathscr{P}_{*}[27$. It is apparent that the 


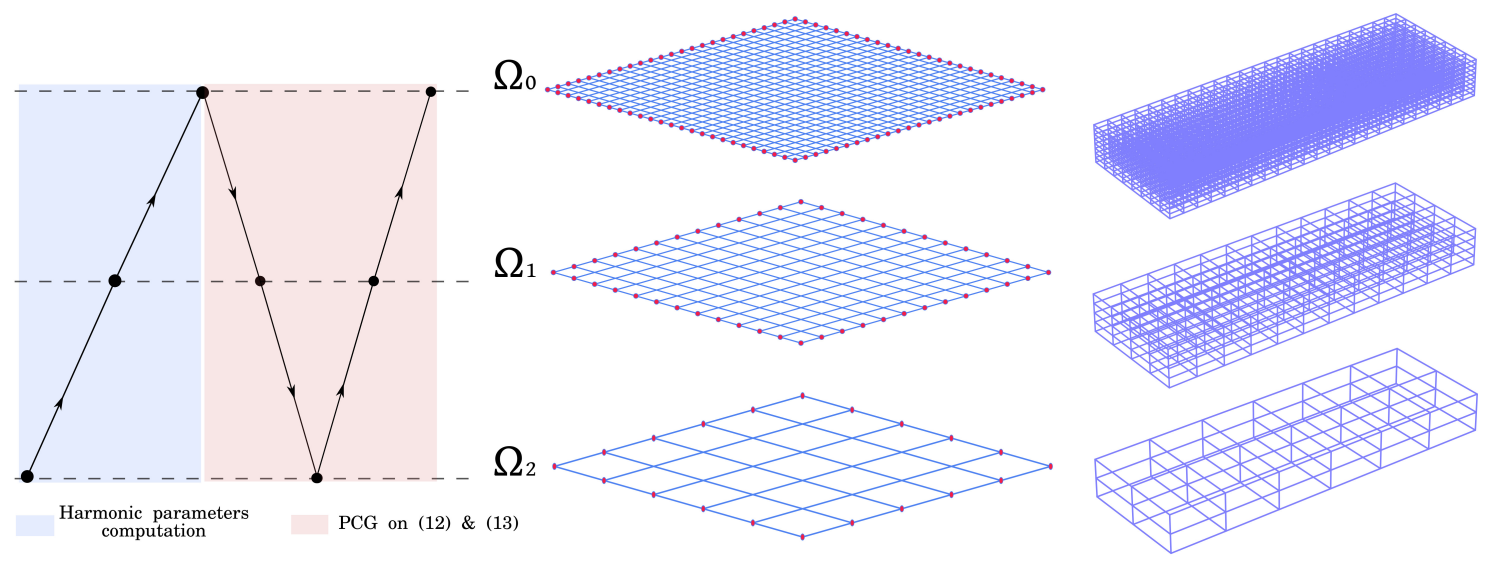

Figure 3: Sketch of the multilevel procedure for the solution of the state equation, for the particular case of $m=2$ coarsening levels. The picture also show qualitatively the coarsening of $2 \mathrm{D}$ and $3 \mathrm{D}$ structured grids

surrogation is more effective when the remainder term $\varrho$ is small, and therefore $\psi_{l}$ is a close approximation to $\phi_{1}$. The ideal case, producing a decoupled FRF, would be $\psi_{l}=\phi_{1}$. This is however not convenient, taking us back to the accurate solution of the eigenvalue problem. We only aim at computing an approximation of it and a (possibly tight) lower bound $\lambda_{l b}<\lambda_{1}$. The latter will give an upper bound for the excitation frequency, so that with $\sigma \in\left[0, \lambda_{l b}\right], S$ is positive definite and (5) is quadratic.

Moreover, since the modal features evolve with the structural changes, the pair $\left(\psi_{l}, \sigma\right)$ should be updated during the optimization. Doing this we can assure that $\psi_{l}$ is always a good approximation of the current modal shape, and $\sigma$ is close to the frequency peak.

\section{Multilevel solution of the state equation}

At each optimization step the linear system (4) is solved by a Preconditioned Conjugate Gradient (PCG) method, where preconditioning is achieved by a multigrid V-cycle [33. The same set of nested grids used for it can also be exploited for cheaply computing the harmonic parameters. In short, the idea is to directly solve the eigenvalue problem only on a coarse mesh and then to use the projection of the eigenpairs as approximations of the harmonic parameters on finer scales.

Besides $\Omega_{0}$, the discretization where $\mathscr{P}$ and $\mathscr{P}_{*}$ are originally formulated, we consider $(m-1)$ auxiliary discretizations $\Omega_{j}$, obtained by geometrical coarsening, such that $h_{j}=2^{j} h$. We may refer to $\Omega_{0}$ as the fine level, and to $\Omega_{m}$ as the coarse level. For $j>0$ we denote vectors and matrices defined on $\Omega_{j}$ by the corresponding apex, and inter-grid operations are performed by the prolongation $I_{j+1}^{j}$ and restriction $I_{j}^{j+1}$ operators. Here, $I_{j}^{j+1}$ is the linear interpolation operator from $\Omega_{j+1}$ to $\Omega_{j}$ and $I_{j}^{j+1}$ is the full weighting operator (see [34, 33] for details and implementation).

We refer to Figure 3 for a sketch of the procedure. We start solving the eigenvalue equation restricted to $\Omega_{m}$, computing $\left(\lambda_{i}^{m}, \phi_{i}^{m}\right)$ such that

$$
\left(K^{m}-\lambda^{m} M^{m}\right) \phi^{m}=0, \quad \phi^{m} \neq 0
$$


We emphasize that $\left(\lambda_{i}^{m}, \phi_{i}^{m}\right)$ are the only exact (up to numerical precision) eigenpairs computed during the whole process. The number of eigenpairs we need to compute depends on the expected multiplicity of $\lambda_{1}$, and may be relatively large. However, this requires only a small amount of the computational effort needed for solving the eigenvalue problem on $\Omega_{0}$, due to the rapid reduction of DOFs, especially for 3D problems (see Figure 3).

Let us for now focus only on the fundamental eigenpair $\left(\lambda^{m}, \phi^{m}\right)$, removing the subscripts for brevity. The procedure will be extended to a larger set of eigenpairs in Section 4 . Interpolation of $\phi^{m}$ to the next grid gives the vector $\psi^{m-1}=I_{m}^{m-1} \phi^{m}$ which is a combination of the eigenvectors defined on $\Omega_{m-1}$, usually with dominance of the fundamental one. Setting $\sigma=\lambda^{m}$, in general, the pair $\left(\sigma, \psi^{m-1}\right)$ is not an eigenpair on $\Omega_{m-1}$. Therefore, considering the residual equation

$$
S^{m-1}(\sigma) \psi^{m-1}=r^{m-1}
$$

the vector $\psi^{m-1}$ coincides with the error, since $S^{m-1}$ is not singular. The application of a few smoothing steps to 13 filters out very rapidly the high frequency components of $\psi^{m-1}$, leaving the lower ones essentially untouched. For this we use a damped Jacobi iteration, with damping factor of $2 / 3$, and a number of iterations ranging from 5 to 10 . We may denote this operation as $\tilde{\psi}^{m-1}=\mathcal{J}\left(r^{m-1}\right)$. Given the smoothed vector $\tilde{\psi}^{m-1}$, its Rayleigh quotient $\mathcal{R}\left(\tilde{\psi}^{m-1}\right)$ is used for updating $\sigma$, improving the approximation to the eigenvalue on the current grid (i.e. $\sigma>\tilde{\sigma}>\lambda^{m-1}$ ).

The procedure is repeated on the next level $\Omega_{m-2}$, with the projection $\psi^{m-2}=I_{m-1}^{m-2} \tilde{\psi}^{m-1}$, and so on until $\Omega_{0}$ is reached. Now we have the pair $(\tilde{\sigma}, \tilde{\psi})$, where $\tilde{\psi}$ is a good approximation of $\phi$ and is used to set up the load amplitude $f=M \tilde{\psi}$, and $\tilde{\sigma}$ is an upper bound to $\lambda$, from here on denoted as $\lambda_{u b}$. The fine scale linear system then reads

$$
S(\sigma) u=M \tilde{\psi}
$$

and for the reason discussed in subsection 2.1, as well as for ensuring the convergence of the PCG iteration, we need to select $\sigma \in\left[0, \lambda_{l b}\right]$, where $\lambda_{l b}<\lambda$. A lower bound can be computed using a complementarity technique suggested in [35], requiring the computation of the fine scale vector,

$$
w=K^{-1} r=\left(I-\tilde{\sigma} K^{-1} M\right) \tilde{\psi}
$$

relating the fine scale residual with the distance $\left|\tilde{\sigma}-\lambda_{1}\right| / \lambda_{1}$ (see [35], Theorem 3.4 for details). Solution of (15) can also be efficiently obtained by the PCG method.

The stopping criterion for the PCG iteration is usually based on the norm of the residual

$$
\left\|r_{(k)}\right\| \leq \tau_{1}\|M \tilde{\psi}\|
$$

for a tight value of the tolerance $\tau_{1}$ (e.g. from $10^{-6}$ to $10^{-10}$ ). It has long been recognized [36, 37, 38, that, for the sake of running the optimization, such a stopping criterion results in unduly accurate approximations $u_{(k)}$, with corresponding increase of computational cost. Therefore, we consider an early termination of the PCG iteration, according to the criterion

$$
\left|u_{(k)}^{T} M \tilde{\psi}-u_{(k)}^{T} S u_{(k)}\right| \leq \tau_{2} u_{(k)}^{T} S u_{(k)}
$$

that is a particularization of what was originally suggested in 37 .

To motivate the use of (17) we can look at Figure 4, showing a plot of the quantity $1-s^{T} s_{(k)}$, where $s$ is the sensitivity computed from an accurate $u$ and $s_{(k)}$ the one computed from an $u_{(k)}$ 


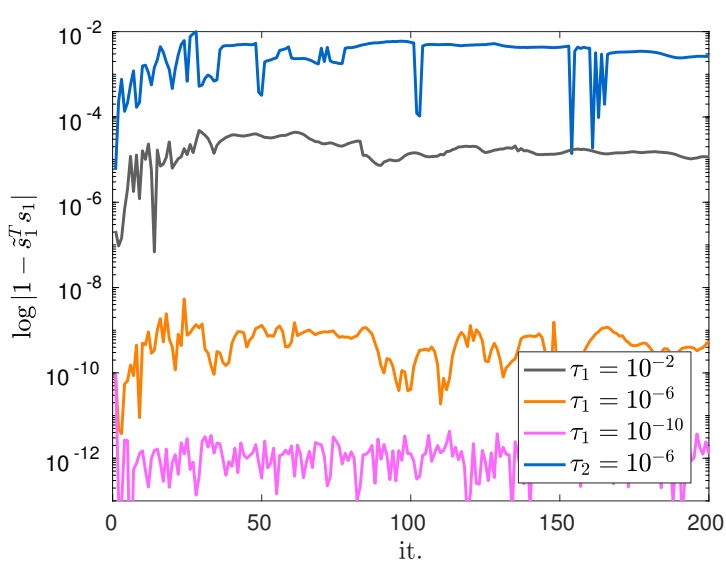

(a)

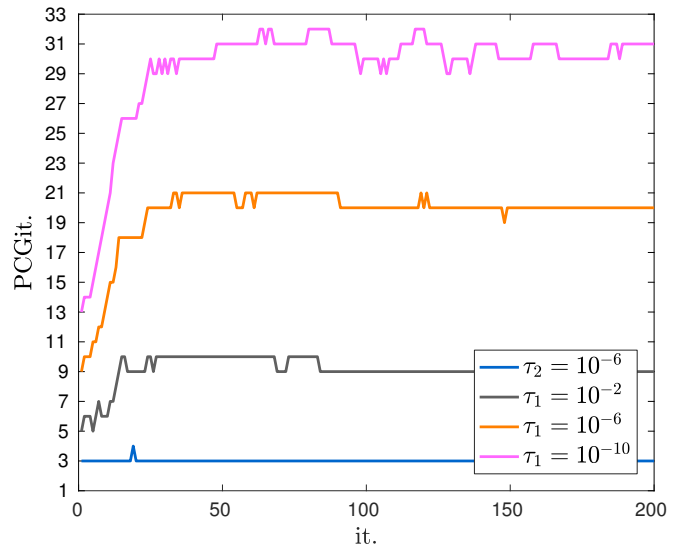

(b)

Figure 4: Accuracy of the sensitivities defined by an approximate solution $u_{(k)}$ satisfying 16 or 17 for a given tolerance $\tau_{1}$ or $\tau_{2}$, repsectively (a), and number of PCG iterations required for meeting the stopping criteria (b)

fulfilling (16) or 17 for a given tolerance. For all $x \in \mathcal{F}_{\text {ad }}$ but stationary points, the smaller this quantity is, the closer $s_{(k)}$ will be to the steepest descent direction. A tight tolerance of $10^{-6}$ or $10^{-10}$ in (16) essentially gives accurate sensitivities, but requires many PCG iterations, as shown in Figure 4 (b). On the other hand, using (17) with $\tau_{2}=10^{-5}$, still gives a sensitivity $s_{(k)}$ differing from the accurate one of less than $1 \%$, which is accurate enough to effectively run the optimization, as we will show in Section 4. We note that only a minimum number of PCG iterations (set to 3) is now required for the presented results.

\subsection{Connections with preconditioned inverse iteration}

The described procedure can be interpreted as a particular application of preconditioned inverse iteration (PInvIt) for solving the eigenvalue problem on $\Omega_{0}$. Given a shift $\sigma$ and a starting vector $u^{(0)}$, inverse iterations [39, 40] involve the solution of a sequence of linear systems

$$
S(\sigma) u^{(\alpha)}=M u^{(\alpha-1)}
$$

When this is achieved by a preconditioned iterative method, a class of inner-outer iterative schemes, known as Preconditioned Inverse Iteration (PInvIt), is obtained 41. The outer step is given by 18 and the inner one is, for example, the PCG iteration computing $u_{(k)}^{(\alpha)}$.

The behaviour of PInvIt has been extensively analyzed in the last decades, with special regard to the influence of inner iteration accuracy on the convergence of the outer one [42, 43, 44, and to the role of the preconditioner [45, 46]. As discussed in [47, 48, provided that $\mathcal{R}^{0}=\mathcal{R}\left(u^{(0)}\right) \in\left(\lambda_{1}, \lambda_{2}\right)$, the sequence $\mathcal{R}^{\alpha}=\mathcal{R}\left(u^{(\alpha)}\right)$ converges at least linearly to $\lambda_{1}$, and this is neither affected by the number of unknowns, nor by preconditioning. This behaviour, along with its matrix-free nature, characterizes PInvIt as the simplest multigrid solver for eigenvalue problems [49].

Furthermore, the initial guess $u^{(0)}=\tilde{\psi}$, computed with little computational effort as described above, gives a very accurate result after a single outer iteration, as we can see from Figure 5 , Starting with e.g. a null vector, three steps of inverse iterations are required to reduce the Rayleigh 


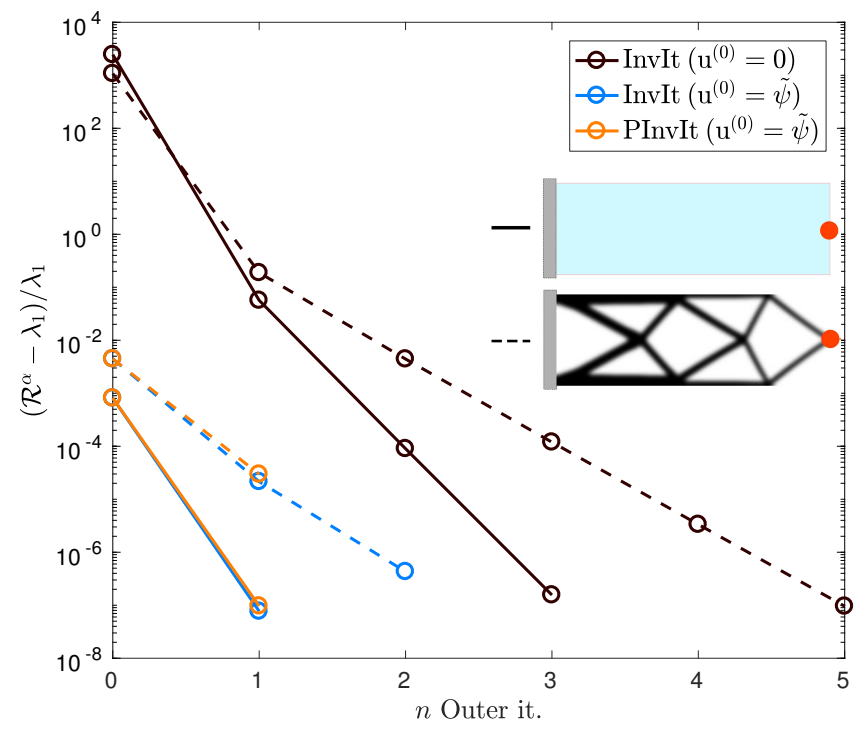

Figure 5: Error reduction for the Rayleigh quotient against the number of outer steps of inverse iterations (InvIt) for the uniform structure and for the optimized one. Black curves refer to application of InvIt with initial guess $u^{(0)}=0$. Blue curves refer to the choice $u^{(0)}=\tilde{\psi}$, computed by the multilevel method and the orange ones take into account the effect of the inexact solution of 18 by the PGC, with the stopping criterion 17 .

quotient error below $10^{-6}$ for the initial structure, and five for the optimized one, with high contrast in material distribution. On the other hand, setting $u^{(0)}=\tilde{\psi}$, after one iteration the error is cut below $10^{-7}$ for the initial structure and to an order of $10^{-5}$ for the optimized one. This allows for a single solution on the fine scale system (14), which is the most expensive part of the procedure. Finally, the orange curves show that, as expected, the effect of preconditioning and inexact solution only marginally affects the error reduction rate.

\section{Extension to multiple load cases}

For the likely situation where eigenvalues become clustered or even repeated and mode switching occurs [11, solving $\mathscr{P}_{*}$ for a single load may results in very poor convergence, or even in evidently non-optimal solutions [27].

When $\lambda_{1}$ becomes, say $q$-repeated, an invariant subspace $\Phi=\left\{\phi_{1}, \ldots, \phi_{q}\right\}$, can be associated with it. A single load vector is likely to become orthogonal to some element of $\Phi$, which will turn into the new fundamental mode. As a consequence, $\lambda_{1}$ will be no more directly excited. Thus, in general we need to consider a set of loads sufficiently large to account for the expected multiplicity of $\lambda_{1}$, and minimize the maximum of the associated dynamic compliances.

The procedure described above can be extended to a preconditioned inverse subspace method 50 , 51, 52 to compute an approximation to the invariant subspace $\Phi$. The general idea for computing the harmonic parameters is essentially unchanged. The whole set $\Psi^{j}=\left\{\psi_{1}^{j}, \ldots, \psi_{q}^{j}\right\}$ is projected through levels, and the smoothing is performed on residuals $R^{j}=\left\{r_{1}^{j}, \ldots, r_{q}^{j}\right\}$, transforming (13) in a block system. We point out that the Rayleigh quotient step for enhancing the upper bound to $\lambda_{1}$, is now replaced by a Ritz projection (still denoted by $\mathcal{R}$ ) in order to ensure that $\Psi^{j}$ is an 
invariant subspace. The Ritz values, collected in the diagonal matrix $\tilde{\Sigma}^{j}=\operatorname{diag}\left\{\tilde{\sigma}_{1}^{j}, \ldots, \tilde{\sigma}_{q}^{j}\right\}$, are upper bounds to the eigenvalues.

On the fine level, the block version of PCG [53, 54 is used for solving the fine level equations (15) and (14), computing the sets $U_{(k)}=\left\{u_{1(k)}, \ldots, u_{q(k)}\right\}$ and $W_{(k)}=\left\{w_{1(k)}, \ldots, w_{q(k)}\right\}$. Again, the iteration is stopped according to (17), applied to each $u_{i(k)}$ and $w_{i(k)}$, respectively.

Considering this more general situation, Algorithm 1 lists the operations involved in the computation of the harmonic load parameters with the multilevel method. This is then inserted within a standard procedure for topology optimization, as listed in Algorithm 2.

As it can be expected, clustering of eigenvalues makes the procedure more delicate. In particular, it cannot be strictly ensured that passing from $\Omega_{j+1}$ to $\Omega_{j}$ the quantity $\sigma=\min _{i} \tilde{\Sigma}^{j+1}$ stays enclosed between $\left(\lambda_{1}^{j}, \lambda_{2}^{j}\right)$. This does not automatically cause the method to fail, since it has been observed that the Rayleigh quotient is still likely to converge to $\lambda_{1}^{j}[48$. However, this circumstance can not be assured theoretically, but only supported by numerical experience. Also the reliability of the lower bound $\lambda_{l b}$ can no longer be strictly guaranteed.

The above issues can be bonded to another delicate point, concerning the selection of the coarse level $\Omega_{m}$. The maximum grid size $h_{m}$ generally depends on the magnitude of $\lambda_{1}$, on the distance $\left(\lambda_{2}-\lambda_{1}\right)$, as well as on the order of finite element approximation and of inter-grid operators [55, 56].

Here we selected the coarse level $\Omega_{m}$ to have a sufficiently accurate description of the structural properties, still substantially reducing the computational effort for the direct eigenvalue solution. In this way we have not experienced fails in convergence even for the examples where $\lambda_{1}$ was repeated. However, we do not claim that the method is completely free from the above mentioned troubles, when applied to more involved problems.
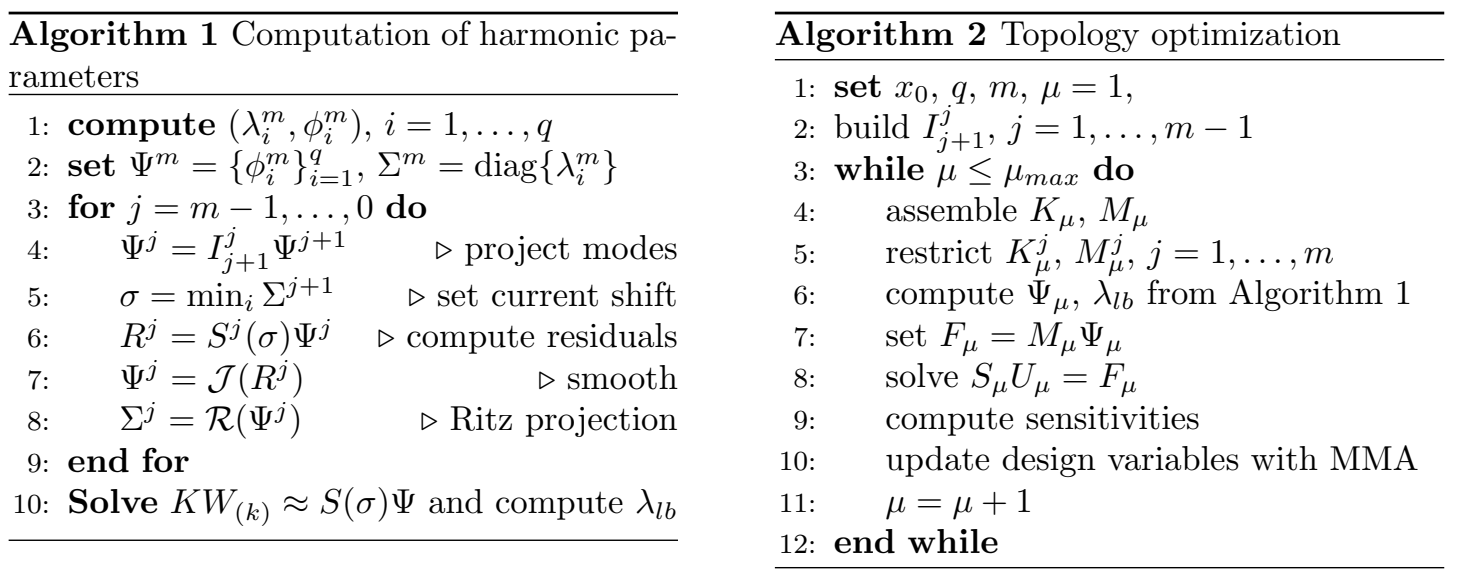

\section{Numerical examples}

We now demonstrate the performance of the proposed approach which has been implemented in Matlab. Besides the 2D cantilever example introduced in Section 2, we consider a 2D example with closely spaced eigenvalues and two 3D beams with bending and torsional fundamental modes, respectively. The material parameters used in (1) are $E_{0}=\rho_{0}=10^{-3}$ and $E_{1}=\rho_{1}=1$, and the penalization is set to $p=4$. The occurrence of spurious vibration modes in regions where $x$ goes to zero is prevented by the relatively high lower bound on stiffness. However, the application to 
higher contrast problems is demonstrated and discussed at the end of Subsection 5.1. The fine discretizations $\Omega_{0}$ are set up with $\left(e_{x}, e_{y}\right)$ bilinear quadrilaterals and $\left(e_{x}, e_{y}, e_{z}\right)$ trilinear hexahedrons for the $3 \mathrm{D}$ example and a convolution filter with radius $\delta_{0}$, scaling with the mesh size, is applied to design variables [57, 58. The eigenvalue equations $(2 \mathrm{~b})$ and $(12)$ are solved with the Matlab function eigs, and the MMA [59] is used for updating the design variables. In this work we are not concerned with a detailed study of the behavior of the optimizer, which can be significantly affected by the asymptotes and step length parameters. We fix these parameters for each example and keep them constant during the whole optimization. We also do not consider a rigorous convergence criterion, but simply run the optimization for a fixed number of steps. In the following, the fine scale eigenvalues obtained while solving $\mathscr{P}$ and $\mathscr{P}_{*}$ are denoted as $\lambda_{i}$ and $\lambda_{i}^{*}$, respectively. We remark that the latter play no role in the proposed approach, and are computed only for the sake of comparison.

\subsection{Cantilever beam with tip non-structural mass}

We consider again the cantilever of Figure 1 (a), with a fine level discretization $\Omega_{0}=(144,48)$ and $\delta_{0}=2 h$. For this example $\lambda_{1}$ is well separated from the other eigenvalues for each $x \in \mathcal{F}_{\text {ad }}$, and therefore we can consider the single load case $f_{1}=M \tilde{\psi}_{1}$. The multilevel scheme is built considering $m=2$ additional grids, with the coarse level $\Omega_{2}=(36,12)$ reducing the DOFs number of about 15 times. The harmonic parameters $\tilde{\psi}_{1}$ and $\lambda_{l b}$ are updated at each optimization step.

The main results are shown in Figure 6 . The direct approach gives a final eigenvalue $\lambda_{1}=8.7586$. $10^{-2}$ and by FRF minimization we obtain, in the same number of iterations, $\lambda_{1}^{*}=8.7699 \cdot 10^{-2}$. Figure 6 (a), referring to the case where (14) is solved for $\sigma=0$, shows that the evolution of the two is almost indistinguishable during the whole optimization. Figure 6 (b) shows the outcome for the case when the shift $\sigma=\lambda_{l b}$ is considered in (14). Although $\lambda_{1}^{*}$ evolves on a different path from $\lambda_{1}$, it ends up again at essentially the same value $\left(\lambda_{1}^{*}=8.7587 \cdot 10^{-2}\right)$. In both cases we observe that $\lambda_{u b}$ is very tight and $\lambda_{l b}$ is reliable. Furthermore, the smoothness of the dynamic compliance minimization curve witnesses the ability of the load vector to continuously adapt to structural changes.

The effectiveness of the load amplitude $\tilde{\psi}_{1}$ can be seen also from Figure 12 (a), showing the magnitude of the first four coefficients $\gamma_{i 1}$, defined in subsection 2.1. For all the optimization steps, the load contribution is almost entirely on $\phi_{1}$ and it is also interesting to note that the smaller contribution is on $\phi_{2}$ which remains a purely axial deformation.

Figure 6 (c)-(d) show a plot of the quantity

$$
\mathcal{D}_{\sigma}=\frac{\sigma-\lambda_{1}}{\lambda_{1}}
$$

measuring the accuracy of fine grid eigenvalue approximations (generically denoted as $\sigma$ ) computable during the multilevel approach. Starting from a coarse level estimate $\left(\lambda^{4 h}\right)$ which may be quite far from the fine grid eigenvalue, the approximation is substantially improved by the multilevel projection, arriving to $\lambda_{u b}$. The PCG iteration on the fine scale further improves the estimate, given by $\mathcal{R}_{k}=\mathcal{R}\left(u_{(k)}\right)$. As expected, the iteration on a shifted system generally leads to a better estimate of the current eigenvalue (see Figure 6(d)).

Keeping the same coarse level, we now consider three refinements of $\Omega_{0}$ and therefore we introduce more projection levels (up to $m=5$ ). The optimization problems $\mathscr{P}$ and $\mathscr{P} *$ are therefore referred to grids $\Omega_{0}^{+}=(288,96), \Omega_{0}^{2+}=(576,192)$ and $\Omega_{0}^{3+}=(1152,384)$ elements. The latter corresponds to 885,810 degrees of freedom and a single spectral analysis on it would take, on average, 


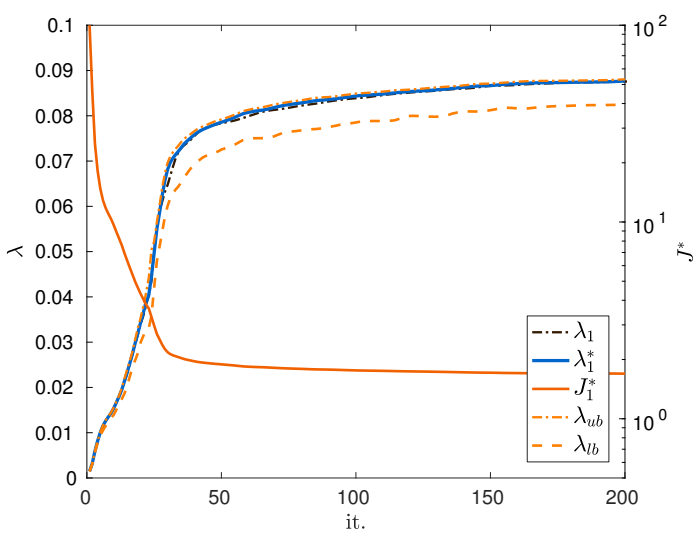

(a) Linear system solved for $\sigma=0$

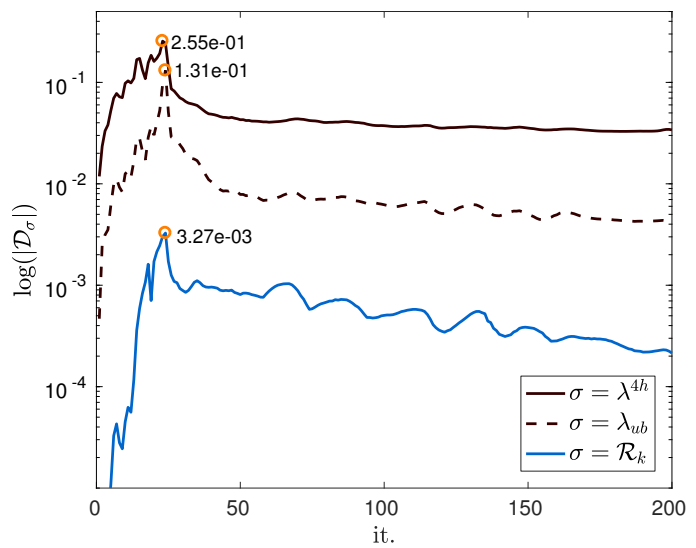

(c) Linear system solved for $\sigma=0$

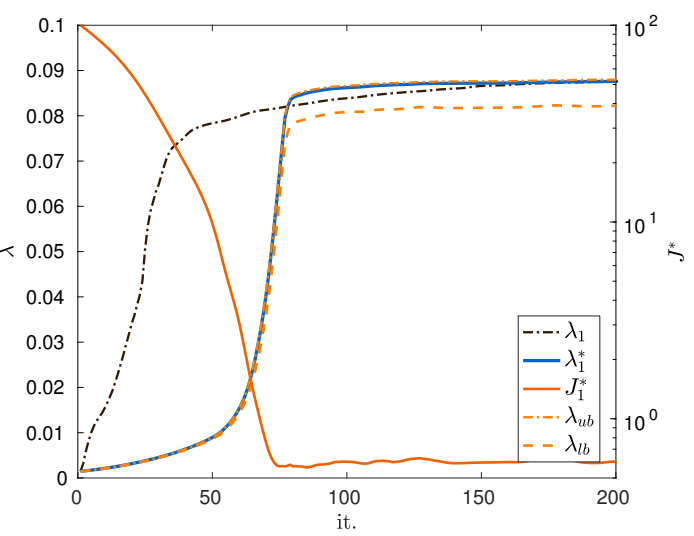

(b) Linear system solved for $\sigma=\lambda_{l b}$

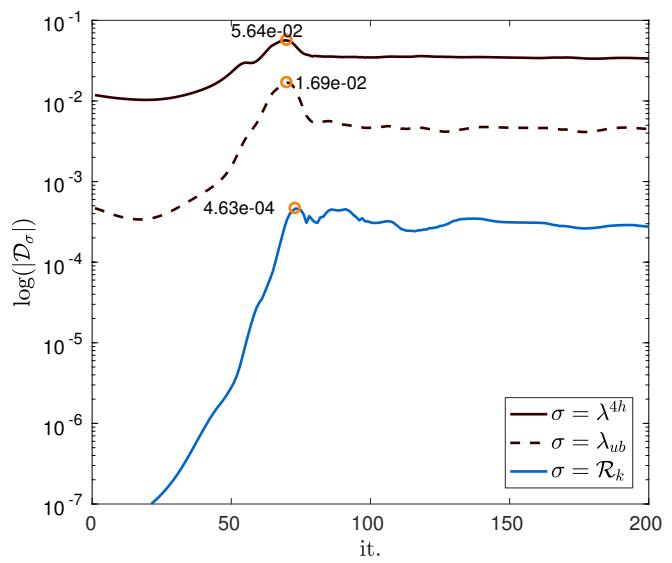

(d) Linear system solved for $\sigma=\lambda_{l b}$

Figure 6: Results of FRF minimization for the 2D cantilever example. (a) and (b) show the evolution of $\lambda_{1}$ and of the computable bounds. Against the right axis is plotted the dynamic compliance minimization history. (c) and (d) show the accuracy, as defined by $(19)$, of some computable eigenvalue approximations 


\begin{tabular}{cccccccc}
$n$ on $\Omega_{0}$ & $\lambda_{1}\left(10^{-2}\right)$ & $\lambda_{1}^{*}\left(10^{-2}\right)$ & r.d. $\left(10^{-3}\right)$ & $\left\|\epsilon_{x}\right\|\left(10^{-2}\right)$ & $T_{\lambda}(\mathrm{s})$ & $T_{*}(\mathrm{~s})$ & $T_{\lambda} / T_{*}$ \\
\hline $14,210(15)$ & 8.75858 & 8.76989 & 1.290 & 1.88 & 74.45 & 16.64 & 4.47 \\
$56,066(58)$ & 8.27404 & 8.22228 & -6.437 & 8.59 & 328.30 & 58.09 & 5.66 \\
$222,722(231)$ & 7.90160 & 7.89473 & -0.870 & 11.17 & 1765.77 & 235.99 & 7.48 \\
$885,810(921)$ & 7.53106 & 7.54480 & 1.825 & 9.75 & 9448.16 & 914.92 & 10.33 \\
\hline
\end{tabular}

Table 1: Results for the 2D cantilever example optimized on different fine levels. In the first column between brackets is the DOFs reduction achieved on the coarse level. The last three columns list the overall computational time for the direct eigenvalues analysis, the one for the proposed multilevel method and respective saving factors
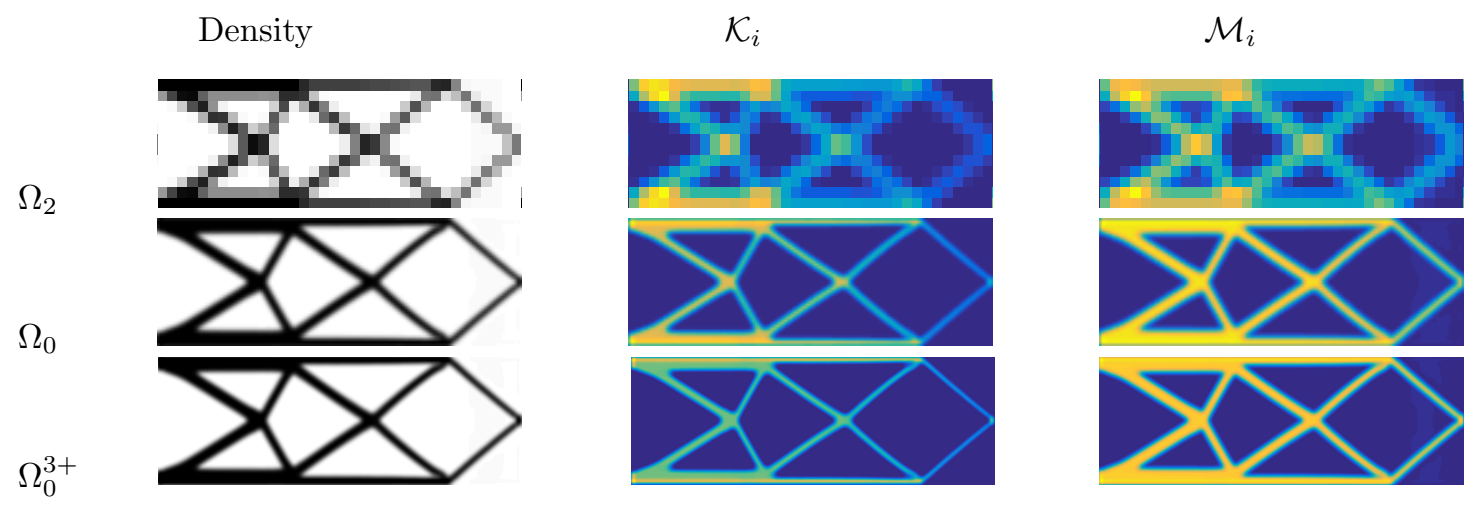

Figure 7: Plot of the physical properties on three scales. The quantities $\mathcal{K}_{i}$ and $\mathcal{M}_{i}$ are normalized with respect to their maximum value

$43 \mathrm{~s}$ and $8.1 \mathrm{~Gb}$ of memory. On the coarse level $\Omega_{0}$, reducing the DOFs number of a factor of about 921 , the spectral analysis takes approximatively $0.42 s$ and $49 \mathrm{Mb}$ of memory.

Table 1 contains the final values of the fundamental eigenvalue, their relative difference and the norm of $\epsilon_{x}=x-x^{*}$, measuring the difference of the two topologies. Figure 7 shows, for three different levels, the distribution of $x^{*}$ and of an isotropic measure of nodal stiffness and mass, $\mathcal{K}_{i}$ and $\mathcal{M}_{i}, i=1, \ldots, n_{\text {nodes }}$. These latter are defined as

$$
\mathcal{K}_{i}=\left(k_{x, i}^{2}+k_{y, i}^{2}\right)^{1 / 2} \quad \mathcal{M}_{i}=\left(m_{x, i}^{2}+m_{y, i}^{2}\right)^{1 / 2}, \quad i=1, \ldots, n_{\text {nodes }}
$$

where $k_{x, i}, k_{y, i}$ and $m_{x, i}, m_{y, i}$ are the diagonal elements of the stiffness and mass matrices associated with the two DOFs of node $i$. From this figure we see how a very rough distribution of the structural properties on the coarse level can be used for obtaining a much more refined design on the fine one.

Considerations about computational savings can be drawn from the last three columns of Table 1 and from Figure 8 . Here $T_{\lambda}$ denotes the overall time spent for spectral analyses while solving $\mathscr{P}$, and $T_{*}$ the one spent for the harmonic analyses with the multilevel approach. The saving factors $\left(T_{\lambda} / T_{*}\right)$ are substantial and, more important, they increase with the problem size, as can be grasped from Figure 8 (a). In this figure we also report the saving factors from the approach of [27], considering an occasional update of the harmonic parameters, and from static compliance optimization, either considering a direct [60] and a multigrid solution [33] of the equilibrium system. The current method is clearly much cheaper than the previous one, even considering a step-by-step update of the harmonic parameters. Moreover, it follows essentially the same trend of the multigrid solution for a static analysis. Finally, from Figure 8 (b) we see the relative cost of the main operations 


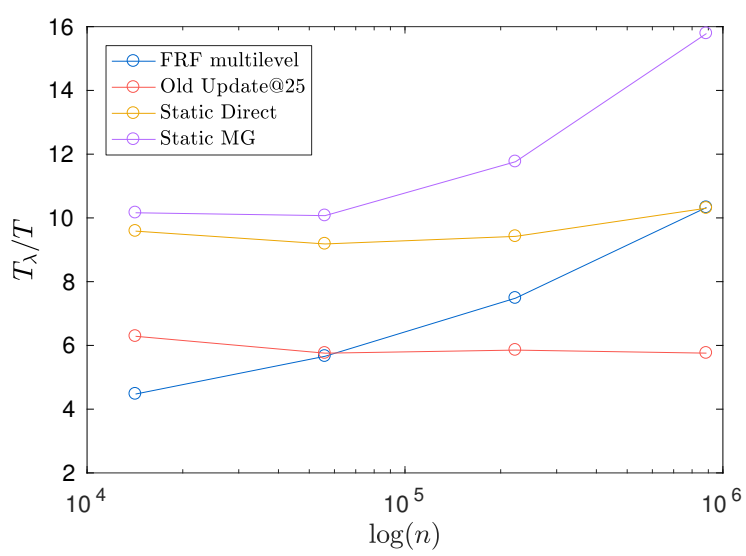

(a)

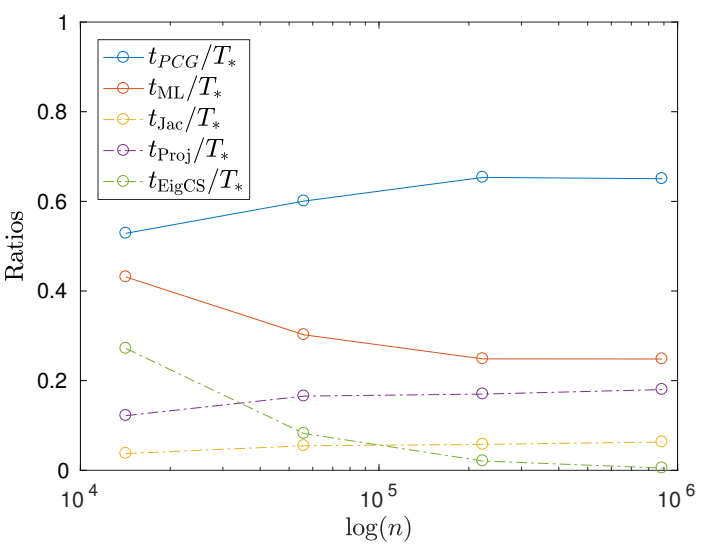

(b)

Figure 8: Saving factors, w.r.t the full eigenvalue approach, for the proposed method and for other procedures, discussed in the text (a). The relative computational cost of the fine grid PCG solutions $\left(t_{\mathrm{PCG}}\right)$, computation of $\tilde{\psi}_{1}$ from multilevel projection $\left(t_{\mathrm{ML}}\right)$, Jacobi smoothing $\left(t_{\mathrm{Jac}}\right)$, projection of the matrices $\left(t_{\mathrm{Proj}}\right)$ and coarse grid eigenvalue solution $\left(t_{\mathrm{EigCS}}\right)$, are shown in (b)

involved in the multilevel procedure. The most expensive part is the solution of the fine scale systems (14)-(15), while the time spent on coarser grids for computing $\tilde{\psi}_{1}$ becomes less and less important, stabilizing (for the present case) to less than $25 \%$ of the total one.

The contrast in material coefficients considered so far was quite low $\left(E_{1} / E_{0}=\rho_{1} / \rho_{0}=10^{3}\right)$. However, the method has also been successfully tested for higher contrasts, a condition which is likely to trigger spurious vibration modes [1, 5, 61. Figure 9 shows the result, for the contrast $E_{1} / E_{0}=\rho_{1} / \rho_{0}=10^{6}$. The convergence curve in Figure 9 (a) shows a sudden dip of the eigenvalue directly computed on the fine scale, in connection with the appearance of small regions of disconnected material, clearly visible from Figure 9 (b), at iteration 73. However, the coarse scale eigenvalue $\left(\lambda_{1}^{C S}\right)$ and all the fine scale approximations derived from it are still smoothly increasing. This is due to the material smearing on the coarse scale, which makes it less sensitive with respect to fine details (see Figure 9 (c)). The final design looks identical to the one in Figure 7 and the final eigenvalue is $\lambda_{1}^{*}=8.81 \cdot 10^{-2}$, slightly higher than before, due to the better material distribution associated with the higher contrast. Of course, for even higher contrast values, the coarse scale may also be affected by spurious modes. In this case, the use of other interpolation schemes than (1) is recommended [14, 62.

\subsection{Example with repeated eigenvalues}

We now test the method on a case where the fundamental eigenvalue is repeated. The geometrical settings are shown in Figure 10 (a). We set $L=1, V^{*}=0.2$ and the magnitude of non-structural mass is $20 \%$ of the structural one. The fine level discretization is $\Omega_{0}=(80,80)$ and $\delta_{0}=2 h$.

The optimized design, obtained after 300 iterations of the direct approach $\mathscr{P}$, is shown in Figure 10 (b). The two lowest eigenvalues of the initial structure are $\lambda_{1}=2.3557 \cdot 10^{-2}$ and $\lambda_{2}=2.3686 \cdot 10^{-2}$ and they remain closely spaced during all the optimization history ending up to the values $\lambda_{1}=0.2969$ and $\lambda_{2}=0.2977$. 

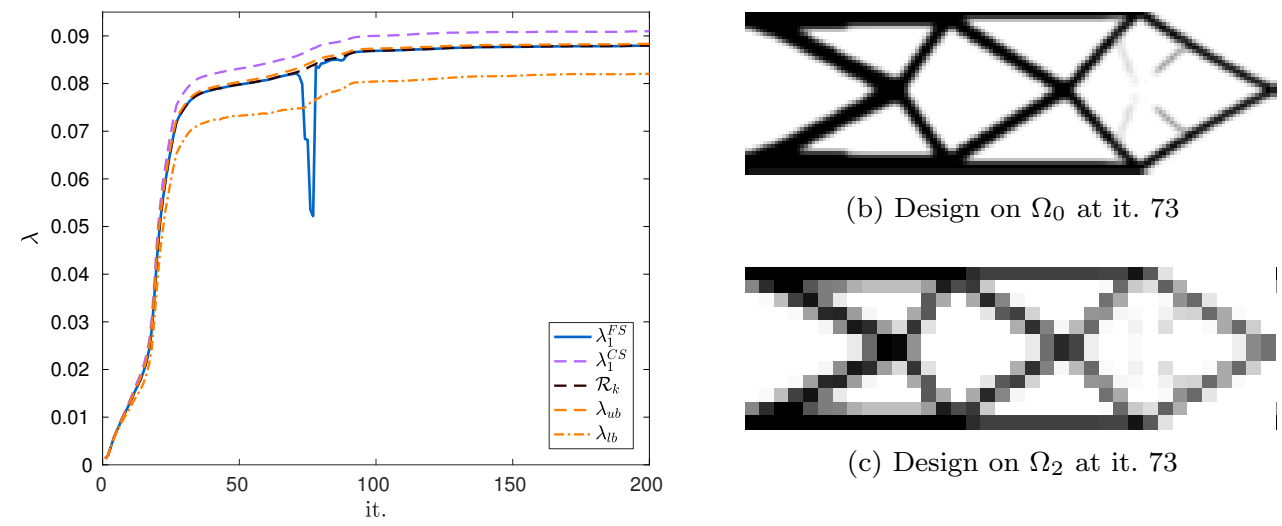

(b) Design on $\Omega_{0}$ at it. 73

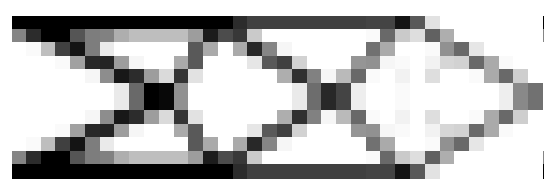

(c) Design on $\Omega_{2}$ at it. 73

(a)

Figure 9: Evolution of the fine scale eigenvalue $\left(\lambda_{1}^{F S}\right)$ and of its approximations computed by the multilevel procedure, for the higher contrast case $E_{1} / E_{0}=\rho_{1} / \rho_{0}=10^{6}$ (a). (b) and (c) show intermediate designs on the fine and coarse scales at iteration 73 , explaining the dip in $\lambda_{1}^{F S}$ in (a)

For this example we must consider at least two independent load vectors in order to ensure that, at each iteration, the eigenmode associated with the lowest eigenvalue is excited. We consider four load vectors, proportional to the amplitudes $\tilde{\psi}_{i}, i=1, \ldots, 4$. These are computed by the multilevel method, that is built again considering $m=2$ coarser levels, with $\Omega_{2}=(20,20)$.

The main results are shown in Figure 11. From Figure 11.(a) we see that after about 30 iterations $J_{1}^{*}$ and $J_{2}^{*}$ become practically coincident and therefore they both drive the optimization. Again, the smoothness of these curves indicates that the load amplitudes properly follow the structural modification. Figure 12 (b) shows the maximum modal forces on $\phi_{1}$ and $\phi_{2}$ for each optimization step, measured as $\max _{l} \gamma_{i l}$, for $i=1,2$. Due to the frequent mode switching, now we do not expect a smooth behaviour of these. However, we observe that, as long as we consider multiple loads, the first two modes are always excited by a high modal force, and therefore the optimization is always effective. The final eigenvalues obtained with $\mathscr{P}_{*}$ are $\lambda_{1}^{*}=0.33247$ and $\lambda_{2}^{*}=0.33401$ and their coalescence can be clearly seen from Figure 11 (b), where the eigenvalues obtained by solving $\mathscr{P}$ are also reported as dashed curves. The result of the direct approach is clearly converging to the one obtained by FRF minimization, but at a slower rate. Therefore, with the fixed number of iterations, for this example we obtained $\lambda_{1}^{*}>\lambda_{1}$. The slower convergence of the direct approach is reflected in the final topologies. The design obtained with the direct approach, Figure 10 (b), is clearly an early stage of the one in (c) and shows some small details and blurred zones which are very rapidly removed during FRF minimization.

In Figure 11 (c) we also see the behaviour of the upper and lower bounds, whose accuracies appear slightly deteriorated compared to the cantilever example, where eigenvalues were well separated. However, they are still reliable in bounding the evolution of $\lambda_{1}^{*}$ and the Rayleigh quotient associated with the fine scale approximation, $\mathcal{R}_{k}$, can still be used as a good approximation of the actual eigenvalue (see Figure 11 (d)).

Similar to the cantilever example previously discussed, here we consider two refinements of the fine level. Problem $\mathscr{P}_{*}$ is formulated on the discretizations $\Omega_{0}^{+}=(160,160)$ and $\Omega_{0}^{2+}=(320,320)$, thus up to $m=5$ projection levels are introduced. $\Omega_{0}^{2+}$ corresponds to $n=206,082$, and on the 


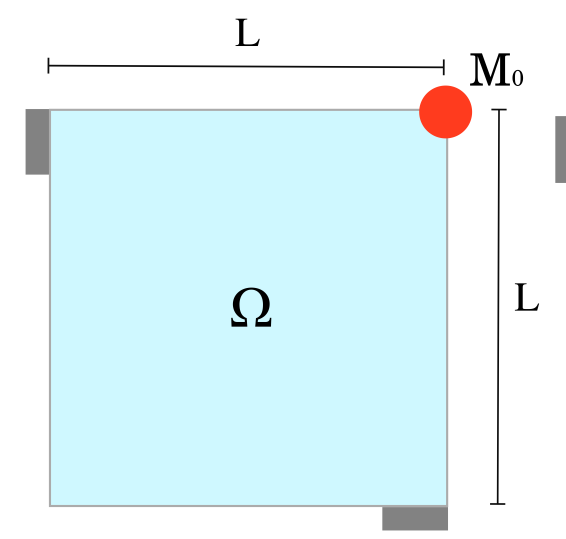

(a)

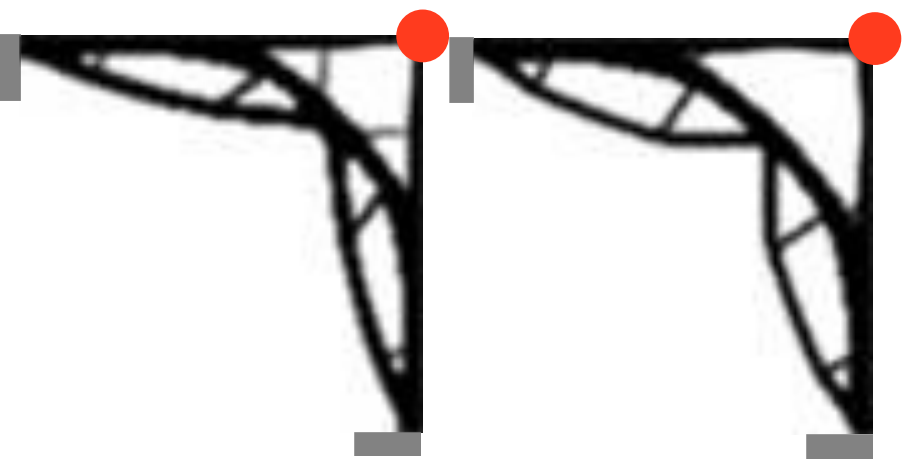

(b) (c)

Figure 10: Geometrical setting (a) a design obtained solving $\mathscr{P}(\mathrm{b})$ and $\mathscr{P}_{*}$ (c) for the structure showing a repeated fundamental eigenvalue.

coarse mesh we achieve a reduction of this number of about 233 times. Even in this case, that is much more sensible to imperfections compared to the cantilever one, due to coalescence of $\lambda_{1}$ and $\lambda_{2}$, an accurate design is obtained on the fine levels (see Figure 13).

\section{3. $3 D$ beams}

We finally provide two moderately large 3D examples. First, we consider the 3D cantilever shown in Figure 14 (a). The non-structural masses are distributed over the nodes of the bottom edge, with a total magnitude $\sum_{i=1}^{e_{z}} M_{0, i}=0.2 \rho V^{*}$, and are active on all the three DOFs. The following fine levels are considered $\Omega_{0}=(96,32,32), \Omega_{0}^{+}=(188,64,64), \Omega_{0}^{2+}=(288,96,96)$ and the filter radius is set to $\delta_{0}=1.2 h$.

We point out that on $\Omega_{0}$, containing $n=316,899 \mathrm{DOFs}$, one direct spectral analysis requires approximatively 20-25 minutes. Eigenvalue problems on $\Omega_{0}^{+}$and $\Omega_{0}^{2+}$, where $n=2,446,275$ and $n=8,157,603$, respectively, clearly leave no chance for a direct solution with a single processor Matlab implementation. We use the coarse level $\Omega_{m}=(24,8,8)$ for problems on $\Omega_{0}$ and $\Omega_{0}^{+}$, and $\Omega_{m}=(18,6,6)$ for the one on $\Omega_{0}^{2+}$, introducing $m=2,3,4$ projection levels, respectively. On these levels, containing few thousands of DOFs, one spectral analysis requires, on average, $0.45-0.58 \mathrm{~s}$. The two lowest eigenvalues of the initial structure are almost coinciding with $\lambda_{1}=1.666 \cdot 10^{-3}$ and $\lambda_{2}=1.685 \cdot 10^{-3}$. Therefore, we considered four independent load vectors in solving $\mathscr{P}_{*}$, accounting for the possible coalescence of higher eigenvalues. The topologies resulting after 100 optimization steps are shown in Figure 14 (b)-(d).

The design corresponding to $\Omega_{0}^{2+}$ is at an earlier convergence stage, due to the huge number of design variables. However, it is clearly converging towards the one obtained on the other levels. For the optimized design the first two eigenvalues, computed on $\Omega_{0}$, are practically coinciding with $\lambda_{1}^{*}=\lambda_{2}^{*}=0.1009$. The compliance minimization history and the curves showing the eigenvalue evolution, along with the upper and lower bounds are shown in Figure 15.

We point out that for this example, considerably more CG iterations (from 8 to 30 ) were needed. This is partly due to the selection of a smaller filter radius, slowing down the error smoothing of 


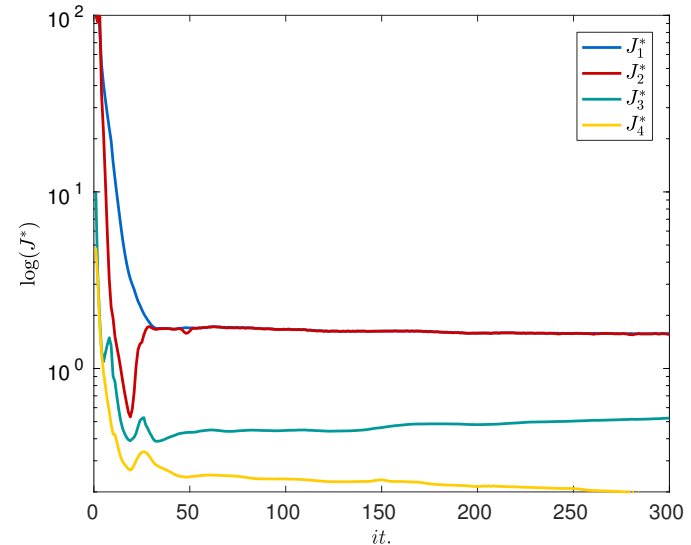

(a)

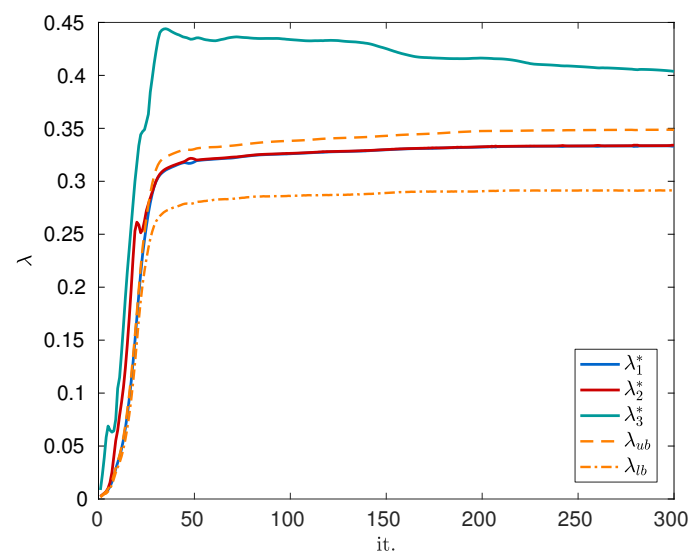

(c)

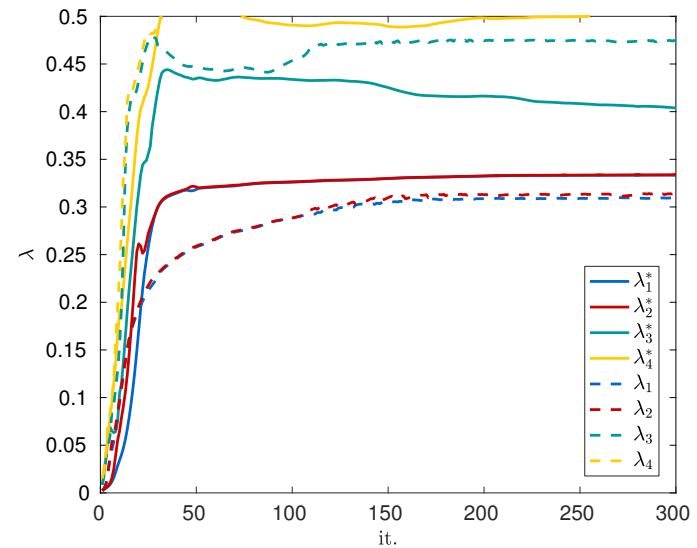

(b)

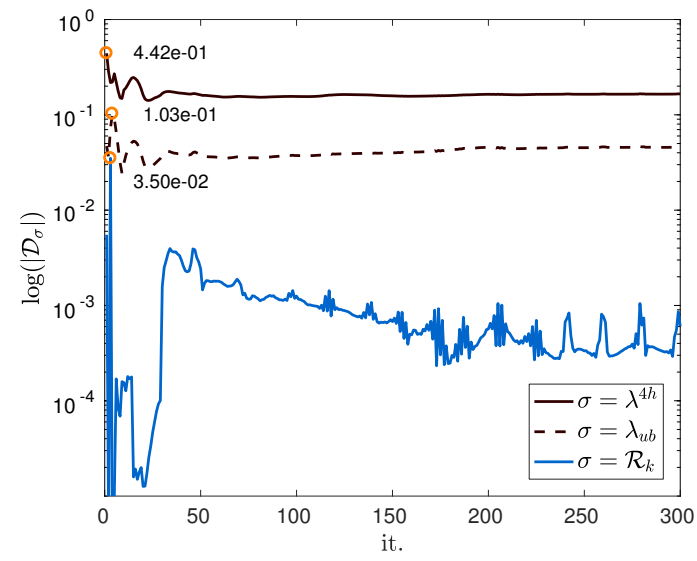

(d)

Figure 11: Results for the case with repeated eigenvalues: (a) dynamic compliance minimization history, (b) evolution curves for the first four eigenvalues, obtained from $\mathscr{P}_{*}$ (continuous curves) and $\mathscr{P}$ (dashed curves), (c) evolution of eigenvalues, together with computable bounds on $\lambda_{1}^{*}$, (d) plot of coefficients $\mathcal{D}_{\sigma}$, defined in 19 


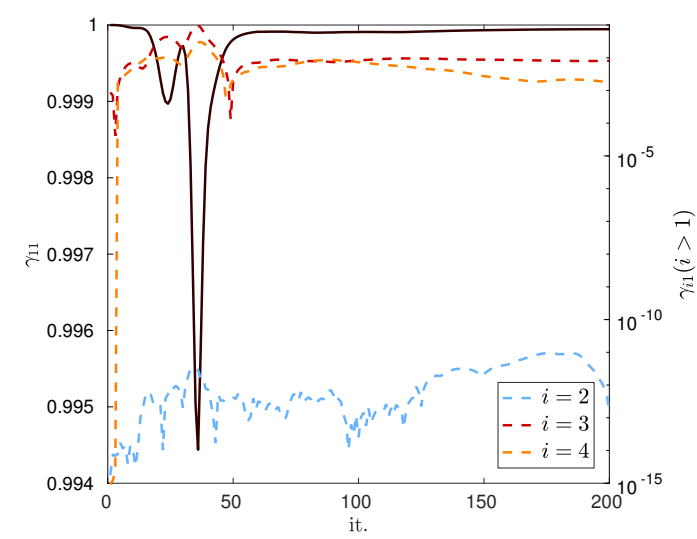

(a)

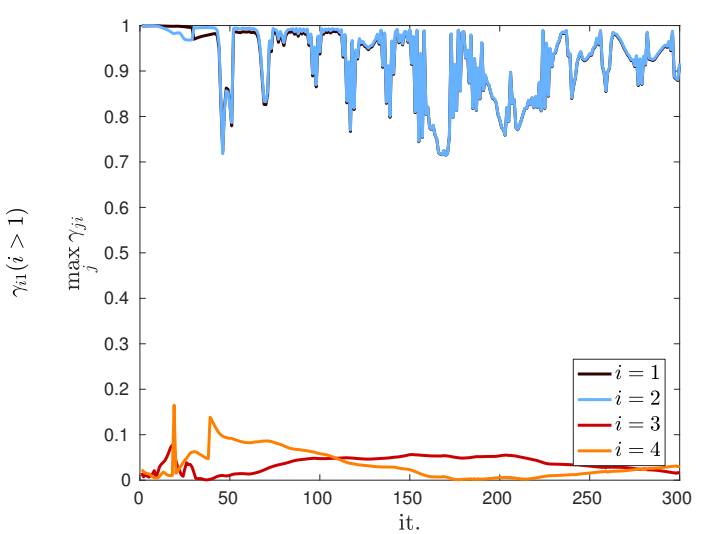

(b)

Figure 12: Plots of coefficients $\gamma_{i l}$, as defined in subsection 2.1 for the 2D cantilever beam example, solved with a single load condition (a) and for the example with repeated eigenvalues (b)

\section{Density}

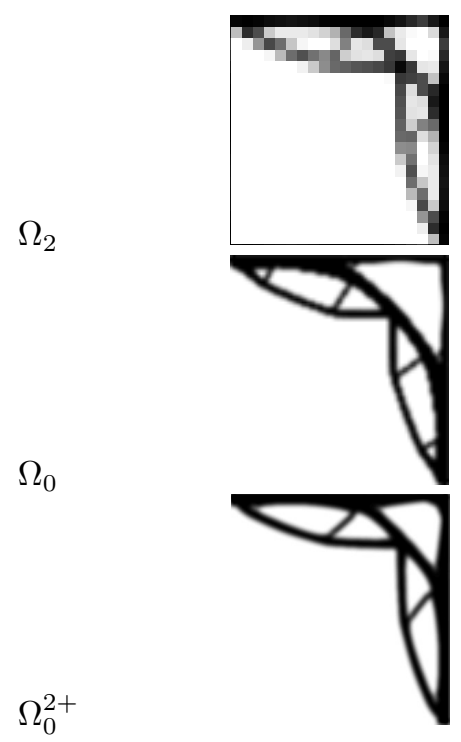

$\mathcal{K}_{i}$
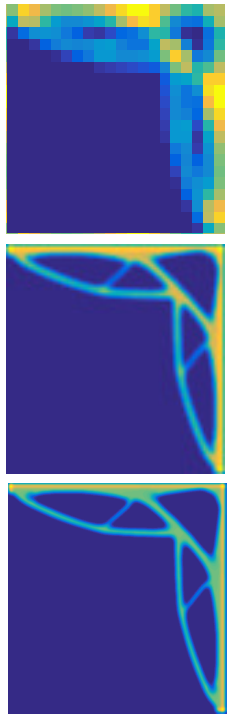

$\mathcal{M}_{i}$
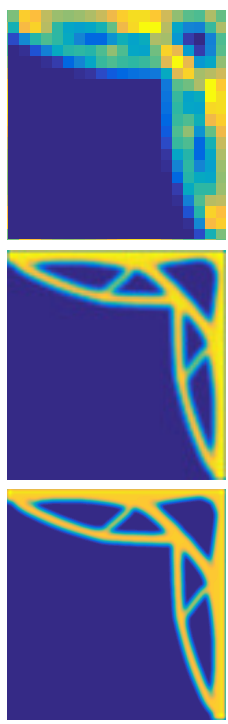

Figure 13: Plot of the physical properties on three scales for the example with a repeated fundamental eigenvalue

the iterative solver [33]. However, their overall number has been observed to be essentially meshindependent. Concerning computational savings, for the fine level $\Omega_{0}$ the proposed method requires about $55 \mathrm{~s}$ of analysis time per iteration. Therefore we estimate a saving factor of more than 20 times, compared to the full eigenvalue solution. For levels $\Omega_{0}^{+}$and $\Omega_{0}^{2+}$, the analysis time is, on average, of 3.9 and 13.5 minutes, and therefore makes possible the solution of the problems within a reasonable amount of time. From the scaling curves reported in Figure 16 we can see how the 


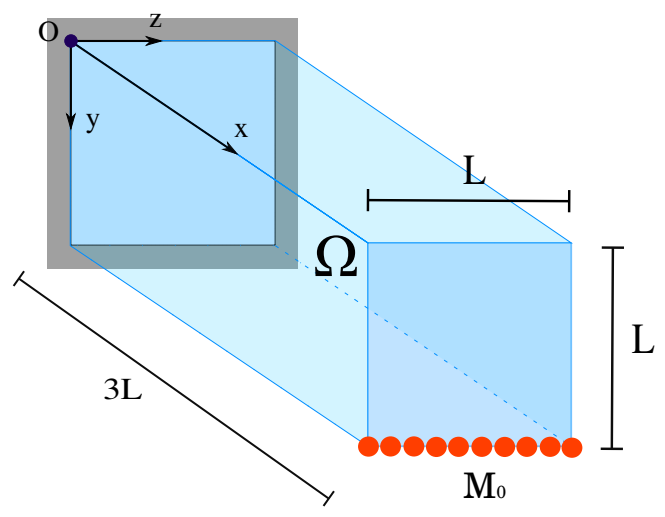

(a)

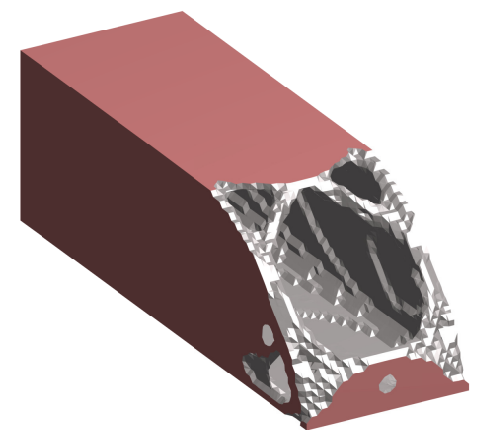

(b) $\Omega_{0}$

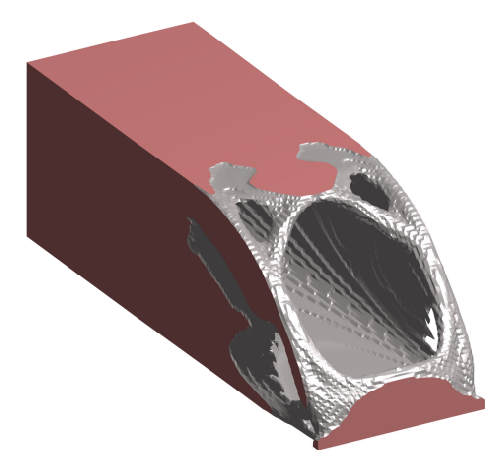

(c) $\Omega_{0}^{+}$

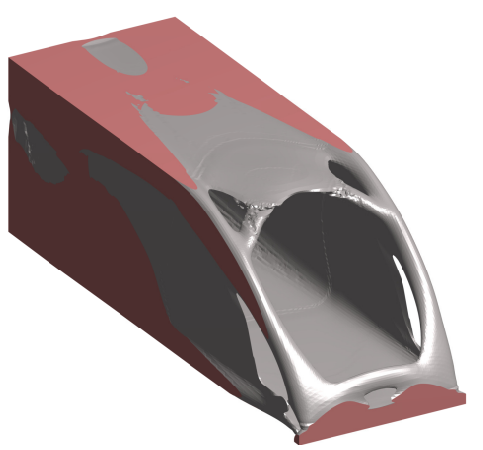

(d) $\Omega_{0}^{2+}$

Figure 14: Geometry for the 3D cantilever beam example (a) and topology optimized on the three fine level considered. The threshold value of 0.8 is adopted for plotting densities in (b) and (c), while 0.6 is used for (d), since the design is at an earlier stage

cost of direct eigenvalue analysis is fast increasing in the 3D case. With the proposed method the complexity is almost linear, both in $2 \mathrm{D}$ and in $3 \mathrm{D}$.

We now refer to the geometry depicted in Figure 17 (a), where elements in the end-cap of the beam $\left(\approx 0.05 e_{x}\right)$ are fixed to be solid. No explicit non-structural masses are considered. Imposing the axial symmetry around $a$, which is the axis passing through the centroid of each $(y, z)$ section, the fundamental mode is forced to be torsional. We therefore model only one quarter of the problem.

We start by considering the discretization $\Omega_{0}=(64,8,8)$ and the coarse level is $\Omega_{1}=(32,4,4)$. The allowed structural volume for the active elements is $V^{*}=0.085$ and the filter radius is $\delta_{0}=\sqrt{2} h$. The design obtained after 500 optimization steps is shown in Figure 17 (b).

Keeping the same coarse scale $\Omega_{1}$ and the same filter radius (i.e. the absolute filter size is now decreasing with mesh refinement), we solve the problem on fine scales $\Omega_{0}^{+}=(128,16,16)$, $\Omega^{2+}=(256,32,32)$ and $\Omega^{3+}=(512,64,64)$. The resulting designs are shown in the first row of Figure 18. As the discretization becomes finer, the optimized topology changes from a truss structure to a closed hollow structure, which is known to be optimal for a rod element subjected to torsion 63. However, it has been recognized that such a configuration is hindered on coarse 


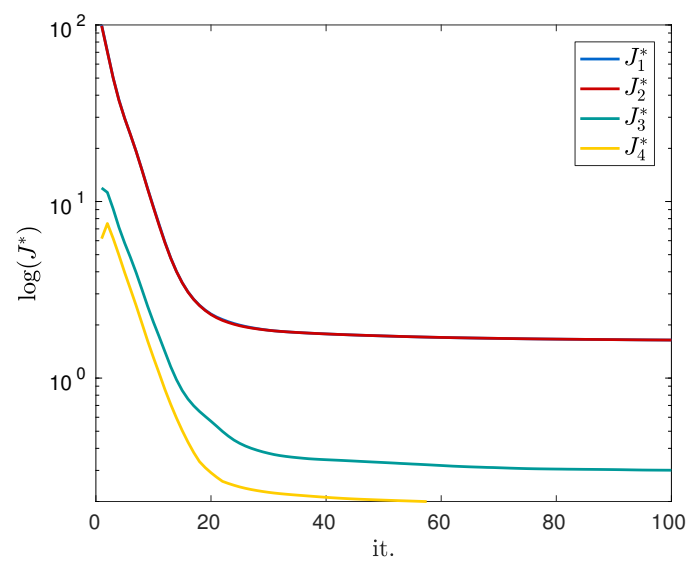

(a)

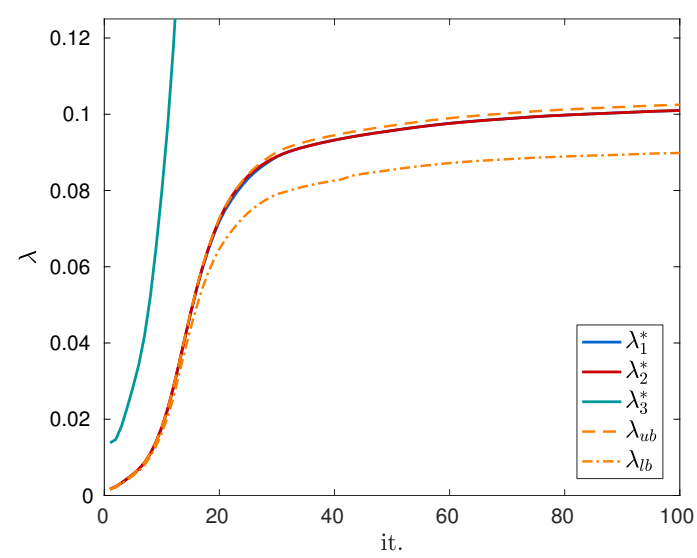

(b)

Figure 15: Results for the 3D cantilever. Compliance minimization history (a) and eigenvalues evolution curves (b). Both these figures show that the first two eigenvalues remain almost coincident during the whole optimization.

meshes [64, 65] (or larger filter sizes), which cannot resolve the small length scale required by the thin-walled true optimum. This finding is further supported by the designs in the second row of Figure 18, which are obtained with a filter radius fixed with respect to the size of the geometry. As expected, we just obtain a refinement of the truss-like design on $\Omega_{0}$.

We can consistently compare the two sets of results by looking at the relative compliance reduction $g_{J}=J_{(\text {initial })}^{*} / J_{(j)}^{*}$ and at the eigenvalue gain $g_{\lambda}=\lambda_{(j)}^{*} / \lambda_{(\text {initial })}^{*}$. These are plotted in Figure 19 (c) and (d), respectively and for the problem solved on $\Omega_{0}$ their final values are $g_{J}=3.98$ and $g_{\lambda}=23.29$. On the three finer grids we obtain $g_{J}=[2.274,1.508,1.409]$ when considering the filter size scaling with the mesh, and $g_{J}=[4.35,4.39,4.93]$ when the filter size is fixed with respect to the geometry. The associated values of the eigenvalue gains are $g_{\lambda}=[41.4,62.3,66.1]$ and $g_{\lambda}=[21.7,21.4,19.4]$, respectively.

This example confirms that fine meshing is needed to resolve pertinent features of optimized $3 \mathrm{D}$ designs. The case considered here result in up to a factor three improvement in objective values compared to coarse scale or length-scale limited designs. However, it also demonstrates the robustness of the proposed approach. Despite the coarse scale solution not being able to resolve the fine scale geometry in this case, the solution procedure and its convergence does not seem to be disturbed.

\section{Concluding discussion}

We have presented a method for solving the eigenvalue topology optimization problem having the same order of complexity as the simpler compliance minimization problem. This has been achieved by resorting to the idea, first proposed in [27, of surrogating the eigenvalue problem by a frequency response one. The computation of the harmonic parameters defining the frequency response problem and its solution procedure have been significantly improved, by introducing a strategy based on a multilevel discretization and iterative solvers. Eigenvalues and eigenvectors are accurately computed only on the coarsest discretization, where the eigenvalue problem can be 


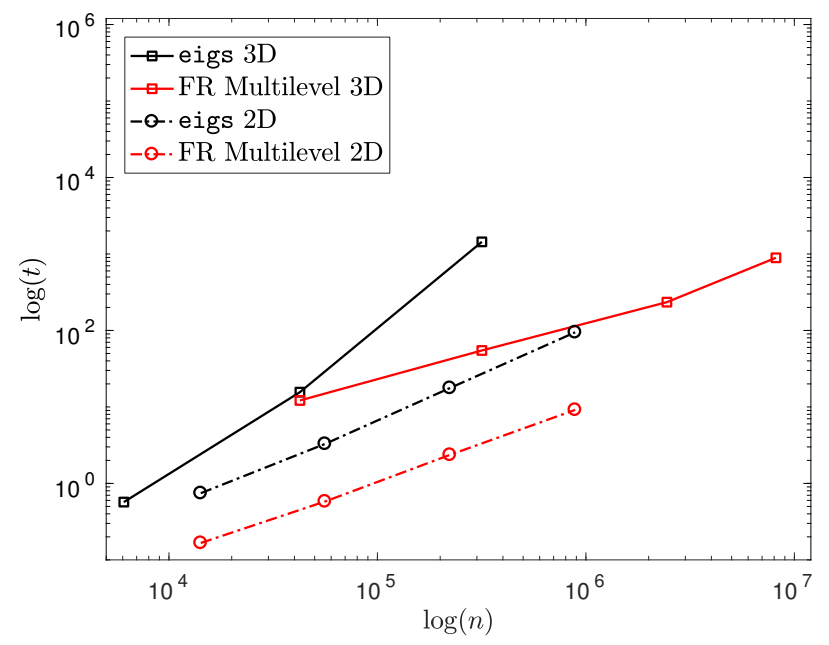

Figure 16: Scaling curves for a single eigenvalue analysis performed by eigs and of a single frequency response analysis with the proposed multilevel procedure, for both the $2 \mathrm{D}$ and the $3 \mathrm{D}$ cantilever example. The slope of the eig curves is approximatively 1.16 in $2 \mathrm{D}$ and 1.98 in 3D. For the the multilevel solution of the FR we have, in both cases, a slope near 1

solved with small computational effort. Then, recovering of the fine scale harmonic loads and the solution of the fine scale linear system only involve cheap operations. This provides the method with multigrid efficiency, meaning that the computational cost scales almost linearly with the dimensions of the fine discretization.

Moderately large scale 2D and 3D applications have been used to prove the feasibility of the method, also for situations where eigenvalues coalesce. Computational savings, compared to direct eigenvalue optimization, are remarkable. For $2 \mathrm{D}$ problems with about $8.6 \cdot 10^{5} \mathrm{DOFs}$, the analysis time is reduced by more than 10 times and for a $3 \mathrm{D}$ problem with about $3.2 \cdot 10^{5} \mathrm{DOFs}$, the saving is of about 20 times. We also solved larger 3D problems, up to $8.1 \cdot 10^{6} \mathrm{DOFs}$, within a reasonable analysis time (about 13.5 minutes per iteration) a task that, within a single-core Matlab implementation, would be impracticable with a direct eigenvalue-based solution. The last example clearly indicates increased reduction of the computational cost for larger problems.

The approach was here tested in a simple Matlab implementation, limiting the testing to moderately large problems. Future studies will include testing the approach in a parallel PETSc-based framework 66] in order to evaluate its potential for solving truly large problems, c.f. up to a billion elements [67. Other future studies will address improvement of the robustness of the method for situations where a large number of eigenvalues are coalescing. We also believe that the method could be made even more efficient, designing different multilevel cycles 28, and by tuning an optimal update strategy for the harmonic parameters. Finally, it should be pointed out that the proposed method applies to unstructured meshes as well. An unstructured mesh can be generated for the coarsest level, and all other levels can be obtained by refinement. The solution procedure, in this case, remains the same as for the structured mesh. On the other hand, for complicated geometries with unstructured mesh representing the finest possible level, construction of geometrybased inter-grid operators might be difficult. The solution procedure in these cases can be built on prolongation and restriction operators based on algebraic multigrid [68]. However, the inter- 


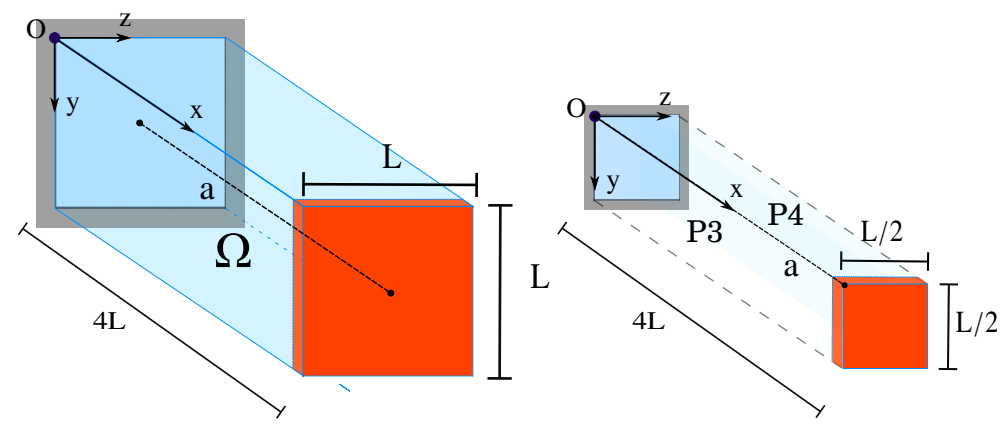

(a)

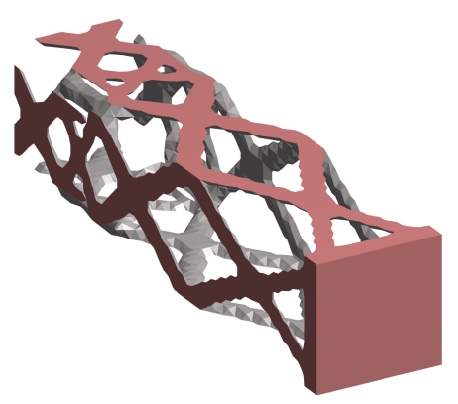

(b)

Figure 17: Geometry for the 3D torsion example, with fixed solid elements at the end-cap (a). The geometry actually modeled is the one on the right, where axial symmetry conditions have been imposed w.r.t. $a$ (i.e. $u_{x}=u_{y}=0$ on plane P3 and $u_{x}=u_{z}=0$ on plane P4). (b) shows the optimized design achieved on $\Omega_{0}$

$$
\Omega_{0}^{+}
$$
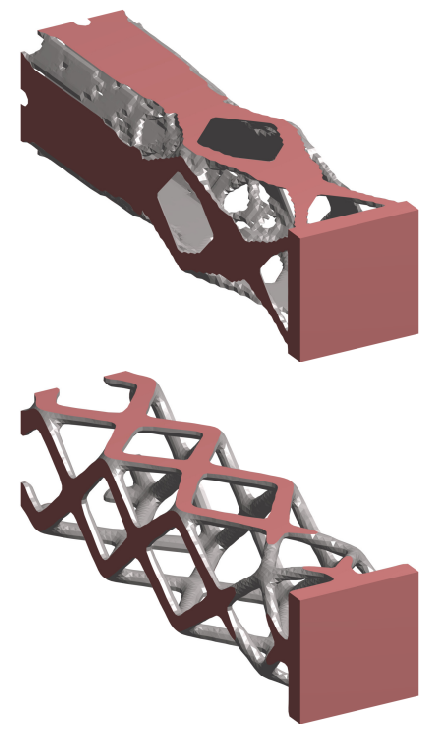

$\Omega_{0}^{2+}$
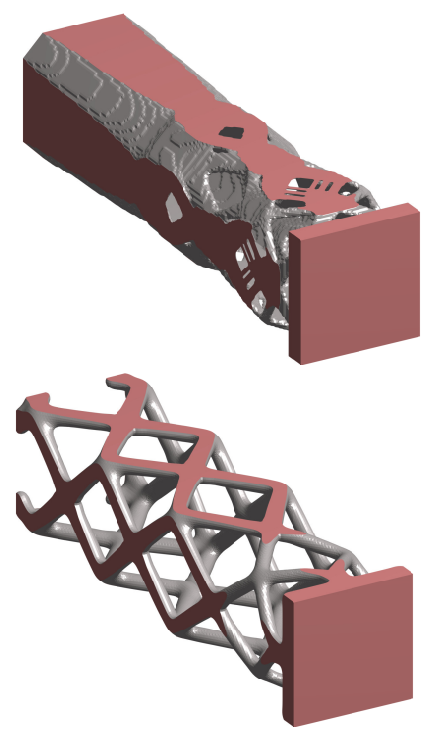

$\Omega_{0}^{3+}$
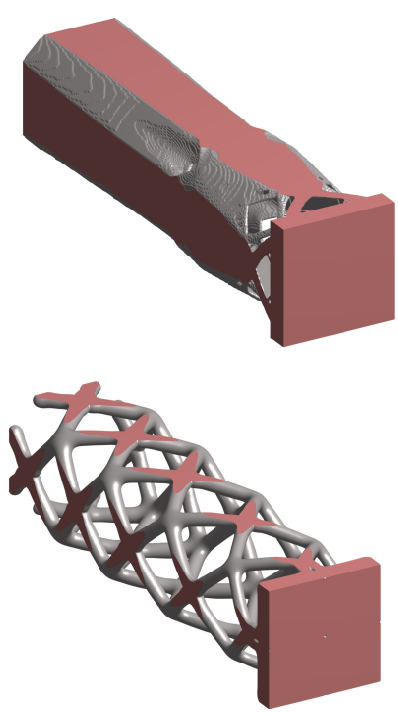

Figure 18: Designs obtained for the torsion example on progressively refined meshes and considering minimal filter radius fixed with respect to element size (top) and fixed with respect to structure size (bottom)

grid operators are usually constructed by using the stiffness matrix and might not provide a good representation of the eigenvalue problem on the coarse solution space. Thus, possible coarsening strategies could follow procedures presented in [69] and utilized for topology optimization in [70]. Such an extension would require further investigations and is left for future research. 


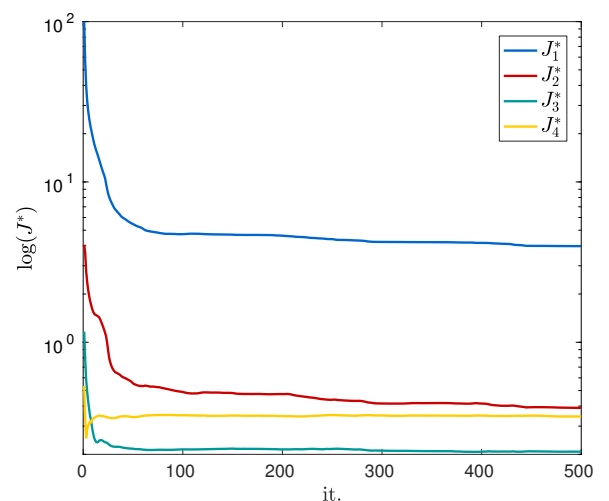

(a)

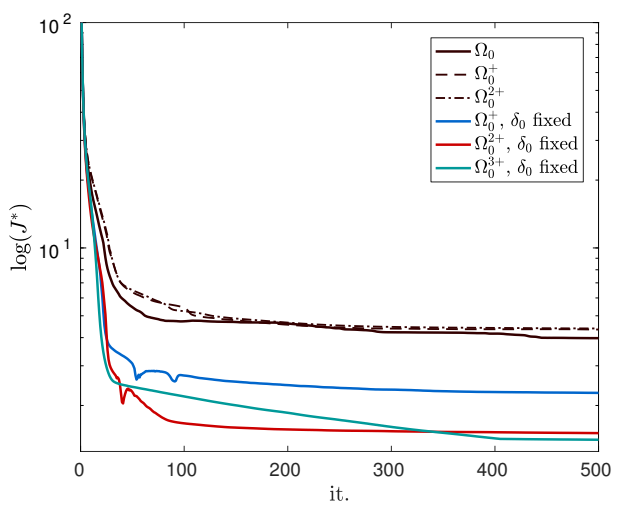

(c) $g_{J}$ value

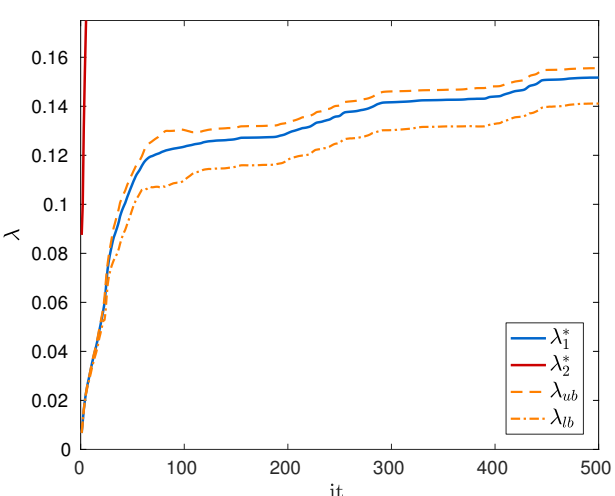

(b)

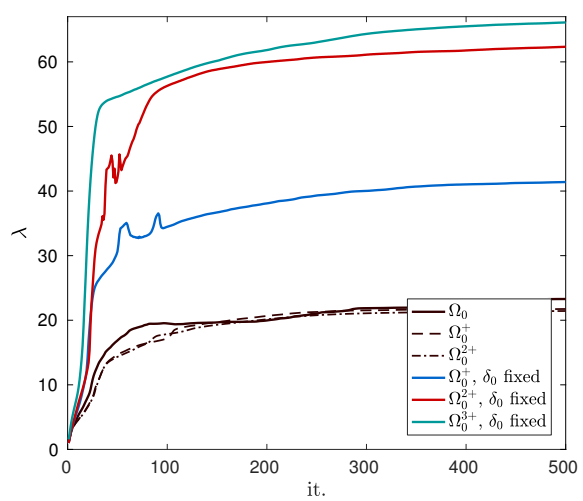

(d) $g_{\lambda}$ value

Figure 19: (a)-(b) Results from the optimization of the 3D torsion example on the discretization $\Omega_{0}$. Compliance reduction ratio $g_{J}(\mathrm{c})$ and eigenvalue gain $g_{\lambda}(\mathrm{d})$ compared for the various meshes, for filters fixed with respect to mesh and geometry ( $\delta_{0}$ fixed), respectively

\section{Acknowledgement}

This project is supported by the Villum Foundation through the Villum Investigator project "InnoTop". The authors are grateful to Prof. Niels Aage and Prof. Oded Amir, for having provided the 3D topology optimization code 33. The first author wishes to thank Jeroen Peter Groen for fruitful discussions on the topic.

\section{References}

[1] M. P. Bendsøe, O. Sigmund, Topology Optimization: Theory, Methods and Applications, Springer, 2004.

[2] A. R. Diaz, N. Kikuchi, Solutions to shape and topology eigenvalue optimization problems using a homogenization method, International Journal for Numerical Methods in Engineering $35(7)$. 
[3] Z. Ma, N. Kikuchi, H. Cheng, Topological design for vibrating structures, Computer Methods in Applied Mechanics and Engineering 121 (1-4) (1995) 259-280.

[4] R. Grandhi, Structural optimization with frequency constraints-A review, AIAA Journal 31 (12) (1993) 2296-2303.

[5] N. Pedersen, Maximization of eigenvalues using topology optimization, Structural and Multidisciplinary Optimization 20 (1) (2000) 2-11.

[6] R. Clough, J. Penzien, Dynamics of structures, McGraw-Hill, 1975.

[7] A. Preumont, Vibration Control of Active Structures: An Introduction, 1st Edition, Solid Mechanics and Its Applications 50, Springer Netherlands, 1997.

[8] O. Sigmund, K. Maute, Topology optimization approaches, Structural and Multidisciplinary Optimization 48 (6) (2013) 1031-1055.

[9] W. Wittrick, On eigenvalues of matrices dependent of a parameter, Numerische Mathematik 6 (1962) 377-387.

[10] E. J. Haug, B. Rousselet, Design sensitivity analysis in structural mechanics II: Eigenvalue variations, Journal of Structural Mechanics 8 (2) (1980) 161-186.

[11] E. J. Haug, K. K. Choi, Systematic occurrence of repeated eigenvalues in structural optimization, Journal of Optimization Theory and Applications 38 (2) (1982) 251-274.

[12] W. Achtziger, M. Kočvara, On the maximization of the fundamental eigenvalue in topology optimization, Structural and Multidisciplinary Optimization 34 (3) (2007) 181-195.

[13] A. P. Seyranian, E. Lund, N. Olhoff, Multiple eigenvalues in structural optimization problems, Structural optimization 8 (4) (1994) 207-227.

[14] S. Jensen, J, N. Pedersen, On maximal eigenfrequency separation in two-material structures: the 1D and 2D scalar cases, Journal of Sound and Vibration 289 (4) (2006) $967-986$.

[15] X. Chen, H. Qi, L. Qi, K.-L. Teo, Smooth convex approximation to the maximum eigenvalue function, Journal of Global Optimization 30 (2) (2004) 253-270.

[16] J. Gravesen, A. Evgrafov, D. M. Nguyen, On the sensitivities of multiple eigenvalues, Structural and Multidisciplinary Optimization 44 (4) (2011) 583-587.

[17] A. J. Torii, J. R. d. Faria, Structural optimization considering smallest magnitude eigenvalues: a smooth approximation, Journal of the Brazilian Society of Mechanical Sciences and Engineering 39 (5) (2017) 1745-1754.

[18] M. Ohsaki, N. Fujisawa, N. Katoh, Y. Kanno, Semi-definite programming for topology optimization of trusses under multiple eigenvalue constraints, Computer Methods in Applied Mechanics and Engineering 180 (1999) 203-217.

[19] P. Zhou, J. Du, Z. Lü, Topology optimization of freely vibrating continuum structures based on nonsmooth optimization, Structural and Multidisciplinary Optimization 56 (3) (2017) 603-618. 
[20] J. S. Arora, Survey of structural reanalysis techniques, Journal of the Structural Division 102 (4) (1976) 783-802.

[21] U. Kirsch, Reanalysis of Structures: A Unified Approach for Linear, Nonlinear, Static and Dynamic Systems, 1st Edition, Solid Mechanics and its Applications, Springer, 2008.

[22] U. Kirsch, A unified reanalysis approach for structural analysis, design, and optimization, Structural and Multidisciplinary Optimization 25 (2) (2003) 67-85.

[23] U. Kirsch, M. Bogomolni, Procedures for approximate eigenproblem reanalysis of structures, International Journal for Numerical Methods in Engineering 60 (12) (2004) 1969-1986.

[24] U. Kirsch, M. Bogomolni, I. Sheinman, Efficient dynamic reanalysis of structures, Journal of Structural Engineering 133 (3) (2007) 440-448.

[25] O. Amir, M. P. Bendsøe, O. Sigmund, Approximate reanalysis in topology optimization, International Journal for Numerical Methods in Engineering 78 (12) (2009) 1474-1491.

[26] M. Bogomolny, Topology optimization for free vibrations using combined approximations, International Journal for Numerical Methods in Engineering 82 (5) (2010) 617-636.

[27] E. Andreassen, F. Ferrari, O. Sigmund, A. R. Diaz, Frequency response as a surrogate eigenvalue problem in topology optimization, International Journal for Numerical Methods in Engineering 113 (8) (2017) 1214-1229.

[28] W. Hackbusch, Multi-grid methods and applications, Springer series in computational mathematics, Springer-Verlag, 1985.

[29] M. P. Bendsøe, O. Sigmund, Material interpolation schemes in topology optimization, Archive of Applied Mechanics 69 (9) (1999) 635-654.

[30] C. Jog, Topology design of structures subjected to periodic loading, Journal of Sound and Vibration 253 (3) (2002) $687-709$.

[31] N. Olhoff, J. Du, Generalized incremental frequency method for topological design of continuum structures for minimum dynamic compliance subject to forced vibration at a prescribed low or high value of the excitation frequency, Structural and Multidisciplinary Optimization 54 (5) (2016) 1113-1141.

[32] A. Quarteroni, R. Sacco, F. Saleri, Numerical Mathematics, Texts in applied mathematics, Springer, 2000.

[33] O. Amir, N. Aage, B. S. Lazarov, On multigrid-CG for efficient topology optimization, Structural and Multidisciplinary Optimization 49 (5) (2014) 815-829.

[34] W. L. Briggs, V. E. Henson, S. F. McCormick, A Multigrid Tutorial (2Nd Ed.), Springer series in computational mathematics, Society for Industrial and Applied Mathematics, Philadelphia, PA, USA, 2000.

[35] I. Šebestovà, T. Vejchodský, Two-sided bounds for eigenvalues of differential operators with applications to Friedrichs, Poincaré, Trace, and similar constants, SIAM Journal on Numerical Analysis 52 (1) (2014) 308-329. 
[36] S. Wang, E. d. Sturler, G. H. Paulino, Large-scale topology optimization using preconditioned Krylov subspace methods with recycling, International Journal for Numerical Methods in Engineering 69 (12) (2007) 2441-2468.

[37] O. Amir, M. Stolpe, O. Sigmund, Efficient use of iterative solvers in nested topology optimization, Structural and Multidisciplinary Optimization 42 (1) (2010) 55-72.

[38] O. Amir, O. Sigmund, On reducing computational effort in topology optimization: how far can we go?, Structural and Multidisciplinary Optimization 44 (1) (2011) 25-29.

[39] J. Wilkinson, The Algebraic Eigenvalue Problem, Monographs on numerical analysis, Clarendon Press, 1988.

[40] I. C. F. Ipsen, Computing an eigenvector with inverse iteration, SIAM Review 39 (2) (1997) $254-291$.

[41] G. H. Golub, Q. Ye, Inexact inverse iteration for generalized eigenvalue problems, BIT Numerical Mathematics 40 (4) (2000) 671-684.

[42] P. Smit, M. Paardekooper, The effects of inexact solvers in algorithms for symmetric eigenvalue problems, Linear Algebra and its Applications 287 (1) (1999) 337 - 357.

[43] J. Berns-Müller, I. G. Graham, A. Spence, Inexact inverse iteration for symmetric matrices, Linear Algebra and its Applications 416 (2) (2006) 389-413.

[44] R. B. Morgan, D. S. Scott, Preconditioning the Lanczos algorithm for sparse symmetric eigenvalue problems, SIAM Journal on Scientific Computing 14 (3) (1993) 585-593.

[45] M. A. Freitag, A. Spence, Convergence of inexact inverse iteration with application to preconditioned iterative solves, BIT Numerical Mathematics 47 (1) (2007) 27-44.

[46] M. A. Freitag, A. Spence, A tuned preconditioner for inexact inverse iteration applied to hermitian eigenvalue problems, IMA Journal of Numerical Analysis 28 (3) (2008) 522.

[47] K. Neymeyr, A geometric theory for preconditioned inverse iteration I: Extrema of the Rayleigh quotient, Linear Algebra and its Applications 322 (1) (2001) 61 - 85.

[48] K. Neymeyr, A geometric theory for preconditioned inverse iteration II: Convergence estimates, Linear Algebra and its Applications 322 (1) (2001) 87 - 104.

[49] K. Neymeyr, Solving mesh eigenproblems with multigrid efficiency, In: Numerical Methods for Scientific Computing. Variational problems and applications (2003).

[50] G. W. Stewart, Simultaneous iteration for computing invariant subspaces of non-hermitian matrices, Numerische Mathematik 25 (2) (1976) 123-136.

[51] M. Robbé, M. Sadkane, A. Spence, Inexact inverse subspace iteration with preconditioning applied to non-hermitian eigenvalue problems, SIAM Journal on Matrix Analysis and Applications 31 (1) (2009) 92-113.

[52] Q. Ye, P. Zhang, Inexact inverse subspace iteration for generalized eigenvalue problems, Linear Algebra and its Applications 434 (7) (2011) 1697 - 1715. 
[53] D. P. O'Leary, The block conjugate gradient algorithm and related methods, Linear Algebra and its Applications 29 (1980) $293-322$.

[54] T. F. Y., D. Owen, D. Peric, A block conjugate gradient method applied to linear systems with multiple right-hand sides, Computer Methods in Applied Mechanics and Engineering 127 (1-4) (1995) 203-215.

[55] L. Banjai, S. Börm, S. Sauter, Fem for elliptic eigenvalue problems: how coarse can the coarsest mesh be chosen? An experimental study, Computing and Visualization in Science 11 (4) (2008) $363-372$.

[56] S. Sauter, $h p$-finite elements for elliptic eigenvalue problems: Error estimates which are explicit with respect to $\lambda, h$, and $p$, SIAM Journal on Numerical Analysis 48 (1) (2010) 95-108.

[57] B. Bourdin, Filters in topology optimization, International Journal for Numerical Methods in Engineering 50 (9) (2001) 2143-2158.

[58] T. E. Bruns, D. A. Tortorelli, Topology optimization of non-linear elastic structures and compliant mechanisms, Computer Methods in Applied Mechanics and Engineering 190 (26) (2001) $3443-3459$.

[59] K. Svanberg, The method of moving asymptotes-a new method for structural optimization, International Journal for Numerical Methods in Engineering 24 (2) (1987) 359-373.

[60] E. Andreassen, A. Clausen, M. Schevenels, B. S. Lazarov, O. Sigmund, Efficient topology optimization in matlab using 88 lines of code, Structural and Multidisciplinary Optimization 43 (1) (2011) 1-16.

[61] D. Tcherniak, Topology optimization of resonating structures using SIMP method, Internal Journal for Numerical Methods in Engineering 54 (11) (2002) 1605-1622.

[62] J. Du, N. Olhoff, Topological design of freely vibrating continuum structures for maximum values of simple and multiple eigenfrequencies and frequency gaps, Structural and Multidisciplinary Optimization 34 (2) (2007) 91-110.

[63] P. Villaggio, Qualitative Methods in Elasticity, Mechanics of Continua, Springer Netherlands, 1977.

[64] C. H. Villanueva, K. Maute, Density and level set-XFEM schemes for topology optimization of 3-D structures, Computational Mechanics 54 (1) (2014) 133-150.

[65] O. Sigmund, N. Aage, E. Andreassen, On the (non-)optimality of Michell structures, Structural and Multidisciplinary Optimization (2016) 361-373.

[66] N. Aage, E. Andreassen, B. Lazarov, Topology optimization using PETSc: An easy-to-use, fully parallel, open source topology optimization framework, Structural and Multidisciplinary Optimization 51 (3) (2015) 565-572.

[67] N. Aage, E. Andreassen, B. Lazarov, O. Sigmund, Giga-voxel computational morphogenesis for structural design, Nature 550 (2017) 84-86.

[68] J. Xu, L. Zikatanov, Algebraic multigrid methods, Acta Numerica 26 (2017) 591-721. 
[69] Y. Efendiev, J. Galvis, X.-H. Wu, Multiscale finite element methods for high-contrast problems using local spectral basis functions, Journal of Computational Physics 230 (4) (2011) 937-955.

[70] J. Alexandersen, B. S. Lazarov, Topology optimisation of manufacturable microstructural details without length scale separation using a spectral coarse basis preconditioner, Computer Methods in Applied Mechanics and Engineering 290 (2015) 156-182. 